\title{
Radiative generation of neutrino masses in a 3-3-1 type model
}

\author{
Arindam Das@, ${ }^{*}$ Kazuki Enomoto๑, ${ }^{\dagger}$ Shinya Kanemura, ${ }^{*}$ and Kei Yagyu ${ }^{\S}$ \\ Department of Physics, Osaka University, Toyonaka, Osaka 560-0043, Japan \\ (Received 13 March 2020; accepted 23 April 2020; published 7 May 2020)
}

\begin{abstract}
A new model for tiny neutrino masses is proposed in the gauge theory of $S U(3)_{C} \otimes S U(3)_{L} \otimes U(1)_{X}$, where neutrino masses are generated via the quantum effect of new particles. In this model, the fermion content is taken to be minimal to realize the gauge anomaly cancellation, while the scalar sector is extended from the minimal 3-3-1 model to have an additional $S U(3)_{L}$ triplet field. After $S U(3)_{L} \otimes U(1)_{X}$ is broken into $S U(2)_{L} \otimes U(1)_{Y}$, the "Zee model" like diagrams are naturally induced, which contain sufficient lepton flavor violating interactions to reproduce current neutrino oscillation data. Furthermore, the remnant $Z_{2}$ symmetry appears after the electroweak symmetry breaking, which guarantees the stability of dark matter. It is confirmed that this model can satisfy current dark matter data. As an important prediction to test this model, production and decays of doubly charged scalar bosons at collider experiments are discussed in successful benchmark scenarios.
\end{abstract}

DOI: 10.1103/PhysRevD.101.095007

\section{INTRODUCTION}

The structure of the electroweak gauge symmetry $S U(2)_{L} \otimes U(1)_{Y}$ has been well established by various experiments. The spontaneous breaking $S U(2)_{L} \otimes$ $U(1)_{Y} \rightarrow U(1)_{\mathrm{em}}$ by the Higgs mechanism has been confirmed by the discovery of the Higgs boson at the LHC. However, the possibility of larger gauge groups including the $S U(2)_{L} \otimes U(1)_{Y}$ symmetry can also be considered at higher energies than the electroweak scale. In fact based on various physics motivations, a plethora of models with extended gauge symmetries has been proposed.

One of the simple but well-motivated extensions is the $S U(3)_{C} \otimes S U(3)_{L} \otimes U(1)_{X} \quad$ (3-3-1) gauge symmetry $[1,2]$. This extension can naturally give the answer to the question in the Standard Model (SM) "Why are there three generations of chiral fermions?" by the gauge anomaly cancellation. Namely, the number of generations has to be the same as the fundamental color degrees of freedom or its multiples [3]. There are many new particles in models with the 3-3-1 gauge symmetry. Clearly, these models necessarily introduce new gauge bosons. Cancellation of the gauge anomaly requires additional

\footnotetext{
*arindam.das@het.phys.sci.osaka-u.ac.jp

†kenomoto@het.phys.sci.osaka-u.ac.jp

*kanemu@het.phys.sci.osaka-u.ac.jp

§yagyu@het.phys.sci.osaka-u.ac.jp
}

Published by the American Physical Society under the terms of the Creative Commons Attribution 4.0 International license. Further distribution of this work must maintain attribution to the author(s) and the published article's title, journal citation, and DOI. Funded by SCOAP. chiral fermions. In order to break the gauge symmetry into the SM one, additional scalar fields have also to be introduced. A next question is whether these new particles can play a role to explain phenomena which cannot be explained in the SM, such as the neutrino oscillation, the existence of dark matter, and the baryon asymmetry of the Universe.

Apart from the 3-3-1 models, a scenario generating tiny neutrino masses at quantum levels can naturally explain their smallness due to loop suppression factors without introducing super heavy new particles. The original model was proposed by Zee [4], in which neutrino masses are generated at the one-loop level. The model proposed by Zee and Babu [5-7] generates neutrino masses at the two-loop level. After these models appeared, models with dark matter particles were also proposed, in which the stability of dark matter is guaranteed by a discrete symmetry which simultaneously forbids tree level diagrams for neutrino masses. For instance, the model proposed by Krauss-NasriTrodden [8] and that by Ma [9] corresponds to those along this line. Therefore, if we can construct models for generating tiny neutrino masses radiatively based on the 3-3-1 scenario, we can explain origins of tiny neutrino masses, dark matter, and generation of chiral fermions simultaneously. This is the subject of the present paper.

Several models with radiative generation of neutrino masses in the 3-3-1 scenario have been proposed in Refs. [10-13]. These models, however, do not contain dark matter candidates. Recently, in Refs. [14,15] one-loop neutrino mass models including a dark matter candidate were constructed by the extension of the minimal model based on the $S U(3)_{C} \otimes S U(3)_{L} \otimes U(1)_{X} \otimes U(1)_{N}$ gauge symmetry, so-called 3-3-1-1 models. In the minimal 
TABLE I. Particle content and charge assignment under the gauge symmetry $S U(3)_{C} \otimes S U(3)_{L} \otimes U(1)_{X}$. The $U(1)^{\prime}$ symmetry is global, which is softly broken by scalar interactions. The flavor indices $i$ and $a$ run over 1-3 and $1-2$, respectively.

\begin{tabular}{|c|c|c|c|c|c|c|c|c|c|c|c|c|c|}
\hline \multirow[b]{2}{*}{ Fields } & \multicolumn{9}{|c|}{ Fermion } & \multicolumn{4}{|c|}{ Scalar } \\
\hline & $L_{L}^{i}$ & $e_{R}^{i}$ & $E_{R}^{i}$ & $Q_{L}^{a}$ & $Q_{L}^{3}$ & $d_{R}^{i}$ & $B_{R}$ & $u_{R}^{i}$ & $U_{R}^{a}$ & $\Phi_{1}$ & $\Phi_{2}$ & $\Phi_{3}$ & $\Phi_{\ell}$ \\
\hline$S U(3)_{C}$ & 1 & 1 & 1 & 3 & 3 & 3 & 3 & 3 & 3 & 1 & 1 & 1 & 1 \\
\hline$S U(3)_{L}$ & 3 & 1 & 1 & $\overline{\mathbf{3}}$ & 3 & 1 & 1 & 1 & 1 & 3 & 3 & 3 & 3 \\
\hline$U(1)_{X}$ & $-2 / 3$ & -1 & -1 & $1 / 3$ & 0 & $-1 / 3$ & $-1 / 3$ & $2 / 3$ & $2 / 3$ & $1 / 3$ & $-2 / 3$ & $1 / 3$ & $4 / 3$ \\
\hline$U(1)^{\prime}$ & 0 & $-q$ & $2 q$ & 0 & 0 & $-q$ & $2 q$ & $q$ & $-2 q$ & $q$ & $-q$ & $-2 q$ & 0 \\
\hline
\end{tabular}

version of 3-3-1-1 models [16-18], three right-handed neutrinos are introduced in order to realize the anomaly cancellation, and tiny masses of active neutrinos are obtained by the seesaw mechanism at tree level. In addition, dark matter candidates are naturally obtained by the remnant discrete symmetry after the spontaneous breaking of the 3-3-1-1 gauge symmetry [16,18]. Scenarios with radiative generation of neutrino masses can also be considered in the framework of 3-3-1-1 models [14,15] by changing $U(1)_{N}$ charges for three right-handed neutrinos to avoid the tree level mass term while keeping the dark matter candidates. However, this model is a bit complicated, because many additional particles are further required for the anomaly cancellation and for making all sterile neutrinos massive [15].

In this paper, we construct a new model with the 3-3-1 gauge symmetry in order to explain tiny neutrino masses, dark matter, and generation of chiral fermions simultaneously. We take the minimal content of fermions required for the gauge anomaly cancellation, while we introduce an additional scalar $S U(3)_{L}$ triplet field to break the lepton number by the scalar self-interactions. Our model then induces "Zee model" like diagrams after the spontaneous breaking of $S U(3)_{L} \otimes U(1)_{X} \rightarrow S U(2)_{L} \otimes U(1)_{Y}$. It has been known that the neutrino oscillation data cannot be reproduced by the Zee model due to a too restricted structure of lepton flavor violating (LFV) interactions, see e.g., $[19,20]$. On the contrary, our model includes additional sources of LFV interactions, so that we can explain current neutrino data.

Another interesting feature of our model is the appearance of an unbroken discrete $Z_{2}$ parity, which guarantees the stability of dark matter candidate, i.e., the lightest neutral particle with a $Z_{2}$-odd charge. This discrete symmetry arises as the remnant symmetry of the 3-3-1 gauge symmetry and a global $U(1)^{\prime}$ symmetry, ${ }^{1}$ where the latter is softly broken and is introduced in order to avoid dangerous flavor changing neutral interactions between a SM fermion and an extra fermion. We find that the $Z_{2}$-odd scalar dark

\footnotetext{
${ }^{1}$ The appearance of such remnant unbroken $Z_{2}$ parity in 3-3-1 models has been pointed out in Refs. [21,22].
}

matter can explain the thermal relic abundance satisfying the current direct search results.

This paper is organized as follows. In Sec. II, we show the particle content in our model, and give the Higgs potential, kinetic terms for scalar fields, and Yukawa interaction terms. In Sec. III, we discuss the generation mechanism for neutrino masses and their mixings. Constraints from LFV decays of charged leptons are considered in Sec. IV. We show numerical results for the correlations between branching ratios of the LFV decays in the parameter sets satisfying the current neutrino data. Section V is devoted to the discussion of the dark matter and the collider phenomenology. We conclude the article in Sec. VI. In the Appendixes, we present stationary conditions for neutral components of scalar fields (Appendix A), the mass formulas of physical scalar bosons (Appendix B) and those for the decay branching ratios for the LFV decays (Appendix C).

\section{MODEL}

In this section, we define our model based on the 3-3-1 gauge symmetry. We first present the particle content and give expressions for component fields of $S U(3)_{L}$ (anti) triplet fields. We then discuss the Higgs potential, kinetic terms for scalar fields, and Yukawa interaction terms in the following subsections in order.

\section{A. Particle content}

The particle content and the charge assignment under the 3-3-1 gauge symmetry are shown in Table I, in which the fermion content is taken to be minimal based on the requirement of the gauge anomaly cancellation. From the cancellation of the pure $S U(3)_{L}$ gauge anomaly, generation must be 3, number of the color, which can be regarded as the origin of the three-generation structure for leptons and quarks [3]. In addition to the gauge symmetry, we introduce a global $U(1)^{\prime}$ symmetry, which is softly broken. This $U(1)^{\prime}$ symmetry is imposed to avoid the dangerous flavor changing neutral current, while it maintains necessary scalar interaction terms in the potential to generate oneloop induced neutrino masses. 
The embedding scheme of leptons and quarks into 3-3-1 multiplets is the same as that given in Ref. [23], in which the electric charge $Q$ is defined by

$$
Q=T_{3}+Y, \quad \text { with } \quad Y=\frac{1}{\sqrt{3}} T_{8}+Q_{X},
$$

where $T_{3}, T_{8}$, and $Q_{X}$ are the third and the eighth components of the $S U(3)_{L}$ generator and the $U(1)_{X}$ charge, respectively. After the 3-3-1 symmetry is broken into the $S U(2)_{L} \otimes U(1)_{Y}$ symmetry, $Y$ in Eq. (1) is identified with the weak hypercharge.

The component fields of left-handed leptons and quarks are then determined as follows:

$$
L_{L}^{i}=\left(\begin{array}{c}
\nu^{i} \\
e^{i} \\
E^{i}
\end{array}\right)_{L}, \quad Q_{L}^{a}=\left(\begin{array}{c}
d^{a} \\
u^{a} \\
U^{a}
\end{array}\right)_{L}, \quad Q_{L}^{3}=\left(\begin{array}{c}
t \\
b \\
B
\end{array}\right)_{L},
$$

where the flavor indices $i$ and $a$ run over 1-3 and 1-2, respectively. In Eq. (2), component fields denoted as lowercase (uppercase) letters can be identified as SM (extra) fermions. We have three, two, and one extra charged leptons, up-type quarks and a down-type quark, respectively, whose electric charges are the same as the corresponding charged leptons and quarks in the SM. All these extra fermions have Dirac mass terms proportional to the vacuum expectation value (VEV) which breaks the $S U(3)_{L} \otimes U(1)_{X}$ symmetry into the $S U(2)_{L} \otimes U(1)_{Y}$ symmetry.

The scalar triplet fields are represented as

$$
\begin{array}{ll}
\Phi_{1}=\left(\begin{array}{c}
\phi_{1}^{+} \\
\phi_{1}^{0} \\
\eta_{1}^{0}
\end{array}\right), \quad \Phi_{2}=\left(\begin{array}{c}
\phi_{2}^{0} \\
\phi_{2}^{-} \\
\eta_{2}^{-}
\end{array}\right), \\
\Phi_{3}=\left(\begin{array}{c}
\eta_{3}^{+} \\
\eta_{3}^{0} \\
\phi_{3}^{0}
\end{array}\right), \quad \Phi_{\ell}=\left(\begin{array}{c}
\eta_{\ell}^{++} \\
\eta_{\ell}^{+} \\
\phi_{\ell}^{+}
\end{array}\right) .
\end{array}
$$

In 3-3-1 gauge theories the Higgs sector contains at least three $S U(3)_{L}$-triplet scalar fields [1,2,23], which give masses to the fermions except for the neutrinos after the electroweak symmetry breaking. In our model, we further introduce an additional triplet field $\Phi_{\ell}$ for the neutrino mass generation, which will be discussed in Sec. III.

\section{B. Higgs potential}

The most general Higgs potential is given by

$$
\begin{aligned}
V= & \sum_{i=1,4} m_{i}^{2}\left|\Phi_{i}\right|^{2}+\left(m_{13}^{2} \Phi_{1}^{\dagger} \Phi_{3}+\epsilon_{\alpha \beta \gamma} \mu \Phi_{1}^{\alpha} \Phi_{2}^{\beta} \Phi_{3}^{\gamma}+\text { H.c. }\right) \\
& +\sum_{i=1,4} \lambda_{i}\left|\Phi_{i}\right|^{4}+\sum_{i, j=1,4}^{j>i}\left(\lambda_{i j}\left|\Phi_{i}\right|^{2}\left|\Phi_{j}\right|^{2}+\rho_{i j}\left|\Phi_{i}^{\dagger} \Phi_{j}\right|^{2}\right) \\
& +\xi_{1}\left(\Phi_{1}^{\dagger} \Phi_{2}\right)\left(\Phi_{3}^{\dagger} \Phi_{\ell}\right)+\xi_{2}\left(\Phi_{2}^{\dagger} \Phi_{3}\right)\left(\Phi_{\ell}^{\dagger} \Phi_{1}\right)+\text { H.c. },
\end{aligned}
$$

where $\Phi_{4}=\Phi_{\ell}$. The $m_{13}^{2}$ and $\mu$ terms softly break the $U(1)^{\prime}$ symmetry, by which the appearance of an additional Nambu Goldstone (NG) boson is avoided. The parameters $m_{13}^{2}, \mu, \xi_{1}$, and $\xi_{2}$ are complex in general, and these complex phases cannot be simultaneously taken to be zero by phase redefinitions of the scalar fields. For simplicity, we take these parameters to be real.

The VEVs of the Higgs triplet fields can generally be taken as

$$
\begin{aligned}
& \left\langle\Phi_{1}\right\rangle=\frac{1}{\sqrt{2}}\left(\begin{array}{c}
0 \\
v_{1} \\
0
\end{array}\right), \quad\left\langle\Phi_{2}\right\rangle=\frac{1}{\sqrt{2}}\left(\begin{array}{c}
v_{2} \\
0 \\
0
\end{array}\right), \\
& \left\langle\Phi_{3}\right\rangle=\frac{1}{\sqrt{2}}\left(\begin{array}{c}
0 \\
v^{\prime} \\
V
\end{array}\right) .
\end{aligned}
$$

The VEV of the third component of $\Phi_{1}$ can be taken to zero without any loss of generality by using the field rotation of $\Phi_{1}$ and $\Phi_{3}$. The VEV $v^{\prime}$ causes phenomenologically dangerous mixing between the SM fermions and the extra ones. Therefore, we arrange $\mu \neq 0$ and $m_{13}^{2}=0$ so as to have a remnant $Z_{2}$ symmetry (denoting it by $\tilde{Z}_{2}$ ), by which $v^{\prime}=0$ is guaranteed and such a dangerous mixing can be avoided. For details, see Appendix A. The charge of the $\tilde{Z}_{2}$ symmetry can be defined as $(-1)^{\left|Q^{\prime}\right| / q}$ with $Q^{\prime}$ being the $U(1)^{\prime}$ charge, by which $\Phi_{1,2}$ and all the SM right-handed fermions are assigned to be odd, while the other fields are even. The $\tilde{Z}_{2}$ symmetry is spontaneously broken by the VEVs $v_{1}$ and $v_{2}$, so that domain walls would appear in the early Universe $[24,25]$. We will briefly comment on this issue at the end of this subsection.

In the following, we assume that $V \gg v_{1}, v_{2}$. Under the setup with $v^{\prime}=0, S U(3)_{L} \otimes U(1)_{X}$ is spontaneously broken into $S U(2)_{L} \otimes U(1)_{Y}$ by the VEV $V$ at higher energy scales than the electroweak scale. Then, the $S U(2)_{L} \otimes U(1)_{Y}$ symmetry is broken down to $U(1)_{\mathrm{em}}$ by $v_{1}$ and $v_{2}$ at the electroweak scale. The Fermi constant $G_{F}$ is reproduced by $G_{F}=\left(\sqrt{2} v^{2}\right)^{-1}$ with $v \equiv \sqrt{v_{1}^{2}+v_{2}^{2}}$. For later convenience, we introduce $\tan \beta=v_{2} / v_{1}$ as the analog of two Higgs doublet models (THDMs).

After the spontaneous breakdown of the 3-3-1 gauge symmetry and the $\tilde{Z}_{2}$ symmetry, another remnant $Z_{2}$ symmetry, let us denote it as $Z_{2}^{\text {rem }}$ appears, whose charge can be defined as $(-1)^{2 s+2 \sqrt{3} T_{8}+\left|Q^{\prime}\right| / q}$ with $s$ being the spin of the particle. In Table II, we show the $Z_{2}^{\text {rem }}$ charges for each particle, where the charges of gauge bosons can be determined from their structures of interactions, see Sec. II C. Because the $Z_{2}^{\text {rem }}$ symmetry is unbroken, the lightest neutral $Z_{2}^{\text {rem }}$-odd particle can be a candidate of dark matter. We will discuss dark matter physics in Sec. V. 
TABLE II. $\quad Z_{2}^{\text {rem }}$ charges of particles. Definitions for the gauge bosons are given in Sec. II C.

\begin{tabular}{llllllllllll}
\hline \hline & \multicolumn{3}{c}{ Fermions } & \multicolumn{3}{c}{ Scalar bosons } & \multicolumn{3}{c}{ Gauge bosons } \\
\hline $\begin{array}{c}Z_{2}^{\text {rem }} \text {-even } \\
\text { fields }\end{array}$ & $e^{i}$ & $u^{i}$ & $d^{i}$ & $\nu_{L}^{i}$ & $\phi_{1,2,3}^{0}$ & $\phi_{1,2, \ell}^{ \pm}$ & & $\gamma^{\mu}$ & $Z^{\mu}$ & $Z^{\prime \mu}$ & $W^{\mu}$ \\
$\begin{array}{c}Z_{2}^{\text {rem }} \text {-odd } \\
\text { fields }\end{array}$ & $E^{i}$ & $U^{a}$ & $B$ & & $\eta_{1,3}^{0}$ & $\eta_{1,2, \ell}^{ \pm}$ & $\eta_{\ell}^{ \pm \pm}$ & $W^{\prime \mu}$ & $Y^{\mu}$ & & \\
\hline \hline
\end{tabular}

Because of the $Z_{2}^{\text {rem }}$ symmetry, we can classify the physical scalar fields into the $Z_{2}^{\text {rem }}$-even and $Z_{2}^{\text {rem }}$-odd ones as follows. In the $Z_{2}^{\text {rem }}$-even sector, we have two pairs of singly charged scalar bosons $H^{ \pm}$and $\hat{H}^{ \pm}$, one $C P$-odd Higgs boson $A$ and three $C P$-even Higgs bosons $H_{i}(i=1$, $2,3)$. The discovered Higgs boson with a mass of about $125 \mathrm{GeV}$ can be identified with the $H_{1}$ state. On the other hand in the $Z_{2}^{\text {rem }}$-odd sector, we have one pair of doubly charged scalar bosons $\eta_{\ell}^{ \pm \pm}$, two pairs of singly charged scalar bosons $\eta^{ \pm}$and $\hat{\eta}^{ \pm}$, and one complex neutral scalar boson $\eta^{0}$. The other eight scalar states are the NG bosons which are absorbed into the longitudinal components of the massive gauge bosons $\left(W_{\mu}, W_{\mu}^{\prime}, Y_{\mu}, Z_{\mu}\right.$, and $\left.Z_{\mu}^{\prime}\right)$, see Sec. II C. In Appendix B, we explicitly show the relation between the mass eigenstates and the weak eigenbasis of the scalar states and their mass formulas.

Let us discuss the effective theory of our 3-3-1 model in the large VEV limit $V \gg v$ with $V \mu \equiv M^{2}$. In this case, the masses of $H^{ \pm}, A$, and $H_{2}$ are determined by the $M$ parameter, ${ }^{2}$ while that of $H_{1}$ is determined by $v$. On the other hand, all the other physical Higgs bosons are decoupled from the theory, as their masses are determined by $V$. Therefore, the scalar sector effectively coincides with a THDM with a special flavor structure which cannot be realized in THDMs with a softly broken $Z_{2}$ symmetry, see Sec. II D. Similar to the usual THDMs, we can define the decoupling limit by $M \gg v$, where only the SM-like Higgs boson $H_{1}$ remains at the scale $v$. We can also define the socalled alignment limit, where the SM-like Higgs boson couplings with the SM gauge bosons and fermions become the same values as those of the SM Higgs boson at tree level. This alignment limit can be taken by choosing potential parameters such that the $(1,2)$ element of the mass matrix of the $C P$-even Higgs bosons given in Eq. (B14) is zero. Therefore, our 3-3-1 model provides another important example that predicts the THDM as the low energy effective theory other than the minimal supersymmetric extension of the SM [26] and composite Higgs models [27-29].

As mentioned in the above, our model potentially has the domain wall problem. It has been known that the energy density of domain walls is only suppressed by the inverse of

\footnotetext{
${ }^{2}$ This parameter plays a similar role to a soft-breaking $Z_{2}$ parameter in THDMs.
}

the radius of the Universe, which is much slower than the dilution of the energy density for ordinary matter and radiation. Therefore, the existence of domain walls could significantly change the history of the Universe. In Refs. [30-33], solutions for the domain wall problem have been discussed. According to Ref. [30], if a discrete symmetry which is spontaneously broken by Higgs VEVs (in our model, this corresponds to the electroweak symmetry breaking VEV $v$ ) is not restored at high temperature, the domain wall problem might not arise. Such a situation can happen if finite temperature effects which are proportional to $T^{2}$ on a negative mass squared term are also negative [34]. In the SM, this does not happen, because there is only one scalar quartic coupling. Such quartic coupling gives a positive effect of finite temperature on the negative mass squared term, so that the broken symmetry at zero temperature is restored at high temperature as it is seen in the usual thermal history of the Universe. On the other hand, if we consider models with multiscalar fields as in our model, this is not always the case, because some combinations of scalar quartic parameters can be taken to be negative so as to realize the symmetry nonrestoration scenario. Thus, we might be able to avoid the domain wall problem. Clearly, more dedicated discussions for this solution have to be done in order to ensure its justification, which is beyond the scope of this paper.

\section{Kinetic terms for scalar fields}

Kinetic terms for the scalar triplet fields are expressed as

$$
\mathcal{L}_{\text {kin }}=\sum_{i=1,4}\left|D_{\mu} \Phi_{i}\right|^{2}
$$

where $\Phi_{4}=\Phi_{\ell}$. The covariant derivative $D_{\mu}$ for $S U(3)_{L}$ triplet fields is given by

$$
D_{\mu}=\partial_{\mu}-i g A_{\mu}-i g_{X} Q_{X} X_{\mu},
$$

with $g$ and $g_{X}$ being the $S U(3)_{L}$ and $U(1)_{X}$ gauge couplings, respectively. The $S U(3)_{L}$ gauge boson $A_{\mu}$ is expressed by the $3 \times 3$ matrix form as

$$
A^{\mu} \equiv A^{A \mu} T^{A}=\left(\begin{array}{ccc}
\frac{1}{2}\left(A_{3}^{\mu}+\frac{A_{8}^{\mu}}{\sqrt{3}}\right) & \frac{A_{12}^{\mu}}{\sqrt{2}} & \frac{A_{45}^{\mu}}{\sqrt{2}} \\
\frac{A_{12}^{* \mu}}{\sqrt{2}} & \frac{1}{2}\left(-A_{3}^{\mu}+\frac{A_{8}^{\mu}}{\sqrt{3}}\right) & \frac{A_{67}^{\mu}}{\sqrt{2}} \\
\frac{A_{45}^{* \mu}}{\sqrt{2}} & \frac{A_{67}^{* \mu}}{\sqrt{2}} & -\frac{A_{8}^{\mu}}{\sqrt{3}}
\end{array}\right),
$$$$
A=1, \ldots, 8,
$$

where we introduced $A_{i j}^{\mu} \equiv\left(A_{i}^{\mu}-i A_{j}^{\mu}\right) / \sqrt{2}$ with $(i, j)=$ $(1,2),(4,5)$, and $(6,7)$. We can identify $W^{\mu} \equiv A_{12}^{\mu}$ and $W^{\prime \mu} \equiv A_{45}^{\mu}$ with the SM $W$ boson and the additional 
charged gauge boson, respectively, while $Y^{\mu} \equiv A_{67}^{\mu}$ with a neutral complex gauge boson. Their masses are given by the VEVs of the Higgs triplet fields as follows:

$$
\begin{aligned}
m_{W} & =\frac{g}{2} v, \quad m_{W^{\prime}}=\frac{g}{2} \sqrt{v^{2} c_{\beta}^{2}+V^{2}}, \\
m_{Y} & =\frac{g}{2} \sqrt{v^{2} s_{\beta}^{2}+V^{2}}
\end{aligned}
$$

where $s_{\theta}=\sin \theta$ and $c_{\theta}=\cos \theta$. Notice here that the gauge bosons $W^{\prime}$ and $Y$ appear in the $(1,3)$ and $(2,3)$ elements of the matrix given in Eq. (8), so that they interact with one $Z_{2}^{\mathrm{rem}}$-even and one $Z_{2}^{\mathrm{rem}}$-odd fermion or scalar bosons in their trilinear interactions. Thus, $W^{\prime}$ and $Y$ can be identified with the $Z_{2}^{\text {rem }}$-odd particles. The other gauge bosons can be identified with the $Z_{2}^{\text {rem }}$-even particles, because they interact with two $Z_{2}^{\text {rem }}$-even or two $Z_{2}^{\text {rem }}$-odd fermions (scalar bosons). This property was already summarized in Table II.

In addition to these complex states, there are three real neutral gauge bosons, where one of them can be identified

$$
\mathcal{M}_{V}^{2}=\frac{V^{2}}{4}\left(\begin{array}{c}
g^{2}\left(1+c_{\beta}^{2} \epsilon\right) \\
\frac{g}{3} \sqrt{3 g^{2}+4 g_{X}^{2}}\left(1-c_{\beta}^{2} \epsilon\right)
\end{array}\right.
$$

with $\epsilon=v^{2} / V^{2}$. In the large $V$ limit, the mass eigenvalues are expressed as

$$
\begin{aligned}
& m_{Z}^{2}=\frac{g^{2} v^{2}}{4}\left[\frac{3 g^{2}+4 g_{X}^{2}}{3 g^{2}+g_{X}^{2}}+\mathcal{O}(\epsilon)\right], \\
& m_{Z^{\prime}}^{2}=\frac{V^{2}}{9}\left[3 g^{2}+g_{X}^{2}+\mathcal{O}(\epsilon)\right] .
\end{aligned}
$$

From the expression of $m_{Z}^{2}$, we see that the weak mixing angle $\theta_{W}$ can be identified with

$$
c_{W} \equiv \cos \theta_{W}=\sqrt{\frac{3 g^{2}+g_{X}^{2}}{3 g^{2}+4 g_{X}^{2}}} .
$$

We then have the same expression of $m_{Z}$ as that of the mass of the SM $Z$ boson. The $U(1)_{\mathrm{em}}$ coupling $e$ is consistently given by $e=g \sin \theta_{W}$ as that in the SM.

The electroweak rho parameter can be expressed at tree level by using the definition of $\theta_{W}$ given in Eq. (13),

$$
\rho=\frac{m_{W}^{2}}{m_{Z}^{2} c_{W}^{2}}=1+\mathcal{O}(\epsilon)
$$

In order to satisfy $|\rho-1| \leq 10^{-3}, V$ has to be taken to be larger than around $8 \mathrm{TeV}$. We note that the calculation of one-loop corrections to the rho parameter is different from that in models with $\rho=1$ at tree level, e.g., the SM. with the massless photon $\gamma^{\mu}$. We can define the basis where $\gamma^{\mu}$ is separated from the other two massive states $\left(\tilde{Z}^{\mu}\right.$ and $\tilde{Z}^{\prime \mu}$ ) as follows:

$$
\begin{aligned}
\left(\begin{array}{l}
A_{3}^{\mu} \\
A_{8}^{\mu} \\
X^{\mu}
\end{array}\right) & =R_{V}\left(\begin{array}{c}
\gamma^{\mu} \\
\tilde{Z}^{\mu} \\
\tilde{Z}^{\prime \mu}
\end{array}\right), \\
R_{V} & =\left(\begin{array}{ccc}
\frac{\sqrt{3} g_{X}}{\sqrt{3 g^{2}+4 g_{X}^{2}}} & \frac{1}{2} & -\frac{3 g}{2 \sqrt{3 g^{2}+4 g_{X}^{2}}} \\
\frac{g_{X}}{\sqrt{3 g^{2}+4 g_{X}^{2}}} & -\frac{\sqrt{3}}{2} & -\frac{\sqrt{3} g}{2 \sqrt{3 g^{2}+4 g_{X}^{2}}} \\
\frac{\sqrt{3} g}{\sqrt{3 g^{2}+4 g_{X}^{2}}} & 0 & \frac{2 g_{X}}{\sqrt{3 g^{2}+4 g_{X}^{2}}}
\end{array}\right) .
\end{aligned}
$$

The $\tilde{Z}$ and $\tilde{Z}^{\prime}$ states can be mixed. Their mass matrix is given by

$$
\left.\begin{array}{c}
\frac{g}{3} \sqrt{3 g^{2}+4 g_{X}^{2}}\left(1-c_{\beta}^{2} \epsilon\right) \\
\frac{1}{9}\left(3 g^{2}+4 g_{X}^{2}\right)\left[1+\left(1+3 s_{\beta}^{2}\right) \epsilon\right]
\end{array}\right),
$$

In models with $\rho \neq 1$ at tree level, the electroweak sector is described by four input parameters which can be chosen to be $\alpha_{\mathrm{em}}, m_{Z}, G_{F}$, and $\delta \rho$ with the last one being the deviation of the rho parameter from unity. This means that the radiative correction to the rho parameter cannot be a prediction, because the additional parameter $\delta \rho$ provides an additional counterterm by which loop corrections to the rho parameter can be absorbed by imposing a renormalization condition. A similar situation can also happen in models with higher isospin scalar multiplets with a nonvanishing VEV such as $S U(2)_{L}$ triplet scalar fields [35,36].

\section{Yukawa interactions}

Thanks to the global $U(1)^{\prime}$ symmetry, Yukawa interaction terms for the SM right-handed fermions and those for the extra right-handed fermions are separately given as follows:

$$
\begin{aligned}
\mathcal{L}_{Y}= & \epsilon_{\alpha \beta \gamma} f_{i j}\left(\overline{L_{L}^{c i}}\right)_{\alpha}\left(L_{L}^{j}\right)_{\beta}\left(\Phi_{\ell}\right)_{\gamma}+y_{e}^{i j} \bar{L}_{L}^{i} \Phi_{1} e_{R}^{j} \\
& +y_{d 2}^{a i} \bar{Q}_{L}^{a} \Phi_{2}^{*} d_{R}^{i}+y_{d 1}^{i} \bar{Q}_{L}^{3} \Phi_{1} d_{R}^{i}+y_{u 1}^{a i} \bar{Q}_{L}^{a} \Phi_{1}^{*} u_{R}^{i} \\
& +y_{u 2}^{a i} \bar{Q}_{L}^{3} \Phi_{2} u_{R}^{i}+y_{E}^{i j} \bar{L}_{L}^{i} \Phi_{3} E_{R}^{j}+y_{D} \bar{Q}_{L}^{3} \Phi_{3} B_{R} \\
& +y_{U}^{a b} \bar{Q}_{L}^{a} \Phi_{3}^{*} U_{R}^{b}+\text { H.c. },
\end{aligned}
$$

where $\epsilon^{\alpha \beta \gamma}$ is the complete antisymmetric tensor with $\alpha, \beta$, and $\gamma$ being the indices for the $S U(3)_{L}$ triplet. Because 
of $e^{\alpha \beta \gamma}$, the complex $3 \times 3$ matrix $f_{i j}$ has also to be antisymmetric.

From the structure of the VEVs and the Yukawa interactions given in Eqs. (5) and (15), respectively, the mass matrices for the SM fermions $\left(M_{f}\right.$ with $\left.f=u, d, e\right)$ and those for the extra fermions $\left(M_{F}\right.$ with $\left.F=U, D, E\right)$ are given by

$$
M_{f}=\frac{v}{\sqrt{2}} Y_{f}, \quad M_{F}=\frac{V}{\sqrt{2}} y_{F},
$$

where

$$
\begin{aligned}
& Y_{e}=y_{e} c_{\beta}, \quad Y_{d}=\left(\begin{array}{lll}
y_{d 2}^{11} s_{\beta} & y_{d 2}^{12} s_{\beta} & y_{d 2}^{13} s_{\beta} \\
y_{d 2}^{21} s_{\beta} & y_{d 2}^{22} s_{\beta} & y_{d 2}^{23} s_{\beta} \\
y_{d 1}^{1} c_{\beta} & y_{d 1}^{2} c_{\beta} & y_{d 1}^{3} c_{\beta}
\end{array}\right), \\
& Y_{u}=\left(\begin{array}{lll}
y_{u 1}^{11} c_{\beta} & y_{u 1}^{12} c_{\beta} & y_{u 1}^{13} c_{\beta} \\
y_{u 1}^{21} c_{\beta} & y_{u 1}^{22} c_{\beta} & y_{u 1}^{23} c_{\beta} \\
y_{u 2}^{1} s_{\beta} & y_{u 2}^{2} s_{\beta} & y_{u 2}^{3} s_{\beta}
\end{array}\right) .
\end{aligned}
$$

We note that $M_{E}, M_{U}$, and $M_{D}$ are respectively the $3 \times 3$, the $2 \times 2$ and the $1 \times 1$ matrices. They are diagonalized by bi-unitary transformations:

$$
f_{L, R}=V_{L, R}^{f} f_{L, R}^{\prime}, \quad F_{L, R}=V_{L, R}^{F} F_{L, R}^{\prime}
$$

As we can see in Eq. (17), the mass matrix for the SM charged leptons has the same form as in the SM; i.e., only one of the Higgs fields gives their masses. On the other hand, the mass matrices for the up-type and the down-type SM quarks are given by two VEVs of the Higgs fields; i.e., $3 \times 3$ matrices are composed of the two independent Yukawa matrices. This structure predicts characteristic flavor-dependent Higgs-boson couplings to quarks [37] in the THDM which is effectively deduced after the

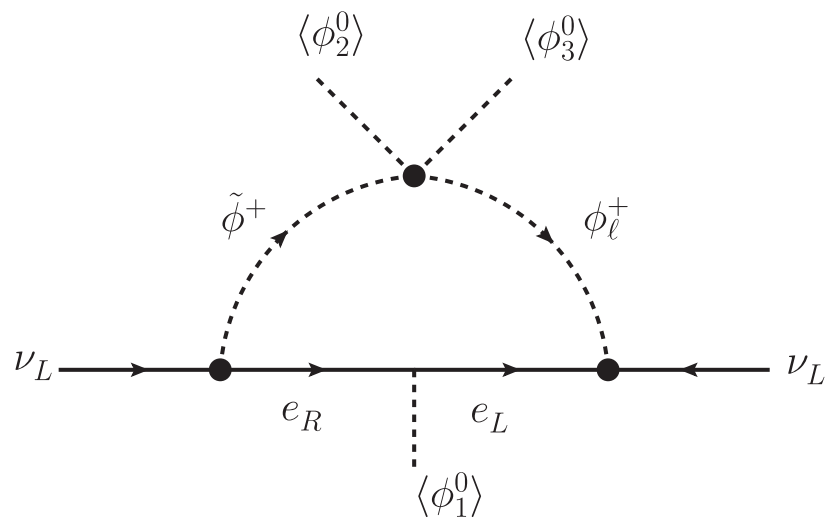

$S U(3)_{L} \otimes U(1)_{X}$ symmetry breaking as discussed in Sec. II B.

The $f_{i j}$ terms in Eq. (15) do not contribute to the masses of charged leptons, but they play an important role for the neutrino mass generation. From these interaction terms, we can assign two units of the lepton number to the Higgs triplet field $\Phi_{\ell}$. This lepton number is explicitly broken by the $\xi_{1}$ and $\xi_{2}$ terms in the Higgs potential given in Eq. (4), and they turn out to be the source of the Majorana masses for the active neutrinos. We will discuss the neutrino mass generation in Sec. III.

\section{NEUTRINO MASSES}

Majorana neutrino masses are generated at the one-loop level as shown in Fig. 1 , in which the $Z_{2}^{\text {rem }}$-even and $Z_{2}^{\text {rem }}$ odd particles run in the loop in the left and right diagram, respectively. We here give relevant interaction terms among physical charged scalar bosons and fermions in their mass eigenbases for the calculation of one-loop induced neutrino masses;

$$
\begin{aligned}
\mathcal{L}_{\text {int }}= & \frac{\sqrt{2}}{V} \frac{s_{\beta}}{\sqrt{1+\frac{v^{2}}{V^{2}} s_{\beta}^{2}}} \bar{\nu}_{L}^{\prime}\left(W M_{E}^{\mathrm{diag}}\right) E_{R}^{\prime}\left(c_{\theta_{\eta}} \eta^{+}-s_{\theta_{\eta}} \hat{\eta}^{+}\right) \\
& -\frac{\sqrt{2} \tan \beta}{v} \bar{\nu}_{L}^{\prime} M_{e}^{\mathrm{diag}} e_{R}^{\prime}\left(c_{\theta_{H}} H^{+}-s_{\theta_{H}} \hat{H}^{+}\right) \\
& -2\left[\bar{\nu}_{L}^{c} F e_{L}^{\prime}\left(s_{\theta_{H}} H^{+}+c_{\theta_{H}} \hat{H}^{+}\right)\right. \\
& -\bar{\nu}_{L}^{\prime c} F W E_{L}^{\prime}\left(s_{\theta_{\eta}} \eta^{+}+c_{\theta_{\eta}} \hat{\eta}^{+}\right) \\
& \left.-\bar{E}_{L}^{\prime c} W^{T} F e_{L}^{\prime} \eta_{\ell}^{++}\right]+ \text {H.c. },
\end{aligned}
$$

where $F \equiv V_{L}^{T} f V_{L}, W=\left(V_{L}^{e}\right)^{\dagger} V_{L}^{E}$, and $\theta_{H}$ and $\theta_{\eta}$ are the mixing angles of the charged scalar fields, see Eq. (B2) and (B16). In the above expression, $M_{e}^{\text {diag }}$ and $M_{E}^{\text {diag }}$ are the diagonalized mass matrices for the SM charged leptons and those for the extra leptons, respectively. The dashed fields are related to the original one by Eq. (18). It is important to

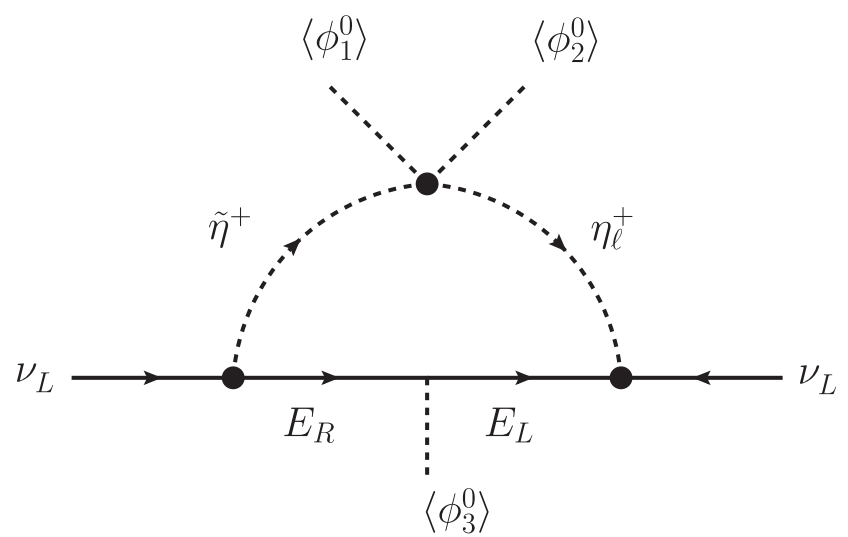

FIG. 1. One-loop diagrams for the neutrino mass generation. Charged scalar fields are written in the basis where the NG boson fields are separated, see Appendix B. 

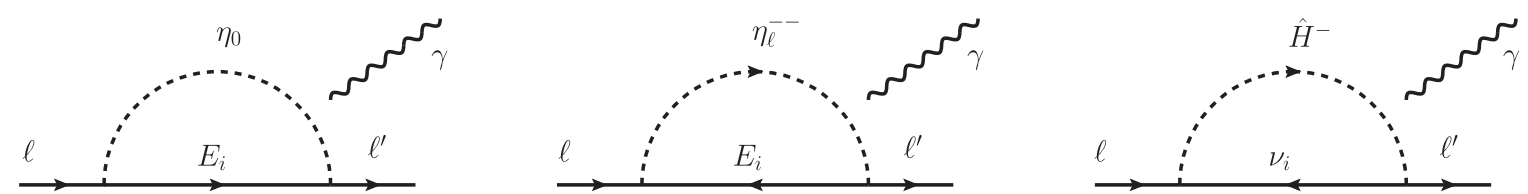

FIG. 2. Diagrams for $\ell \rightarrow \ell^{\prime} \gamma$ processes.

mention here that the appearance of the $W$ matrix which is the $3 \times 3$ unitary matrix similar to the Cabibbo-KobayashiMaskawa matrix plays a crucial role to reproduce the current neutrino oscillation data as it will be clarified below.

The total contribution to the Majorana neutrino masses is then expressed as

$$
\mathcal{M}_{\nu}=\left(\mathcal{M}_{\nu}^{e}+\mathcal{M}_{\nu}^{E}\right)+\left(\mathcal{M}_{\nu}^{e}+\mathcal{M}_{\nu}^{E}\right)^{T},
$$

where $\mathcal{M}_{\nu}^{e}$ and $\mathcal{M}_{\nu}^{E}$ represent the contribution from the left and right diagrams, respectively. They are calculated as

$$
\begin{aligned}
\left(\mathcal{M}_{\nu}^{e}\right)_{i j} & =\frac{C_{e}}{v} F_{i j} m_{j}^{2}, \\
\left(\mathcal{M}_{\nu}^{E}\right)_{i j} & =\frac{C_{E}}{V}(F W)_{i k} M_{k}^{2} G_{k}\left(W^{\dagger}\right)_{k j},
\end{aligned}
$$

where $m_{i} \equiv\left(\boldsymbol{M}_{e}^{\mathrm{diag}}\right)_{i i}$ and $\boldsymbol{M}_{i} \equiv\left(\boldsymbol{M}_{E}^{\mathrm{diag}}\right)_{i i}$, and

$G_{k}=\frac{1}{2} \ln \frac{m_{\eta^{ \pm}}^{2}}{m_{\hat{\eta}^{ \pm}}^{2}}+\frac{M_{k}^{2}+m_{\hat{\eta}^{ \pm}}^{2}}{M_{k}^{2}-m_{\hat{\eta}^{ \pm}}^{2}} \ln \frac{m_{\hat{\eta}^{ \pm}}^{2}}{M_{k}^{2}}-\frac{M_{k}^{2}+m_{\eta^{ \pm}}^{2}}{M_{k}^{2}-m_{\eta^{ \pm}}^{2}} \ln \frac{m_{\eta^{ \pm}}^{2}}{M_{k}^{2}}$.

The flavor independent coefficients $C_{e}$ and $C_{E}$ are given by

$$
C_{e}=\frac{\sqrt{2}}{8 \pi^{2}} \tan \beta c_{\theta_{H}} s_{\theta_{H}} \ln \frac{m_{\hat{H}^{ \pm}}^{2}}{m_{H^{ \pm}}^{2}}, \quad C_{E}=\frac{\sqrt{2}}{8 \pi^{2}} \frac{s_{\beta} c_{\theta_{\eta}} s_{\theta_{\eta}}}{\sqrt{1+\frac{v^{2}}{V^{2}} s_{\beta}^{2}}} .
$$

The mass matrix given in Eq. (20) is diagonalized by introducing the Pontecorvo-Maki-Nakagawa-Sakata matrix $U_{\text {PMNS }}$ as

$$
U_{\mathrm{PMNS}}^{T} \mathcal{M}_{\nu} U_{\mathrm{PMNS}}=\operatorname{diag}\left(m_{\nu}^{1}, m_{\nu}^{2}, m_{\nu}^{3}\right),
$$

where $m_{\nu}^{i}(i=1,2,3)$ are mass eigenvalues for neutrinos, and

$$
\begin{aligned}
U_{\mathrm{PMNS}}= & \left(\begin{array}{ccc}
1 & 0 & 0 \\
0 & c_{23} & s_{23} \\
0 & -s_{23} & c_{23}
\end{array}\right)\left(\begin{array}{ccc}
c_{13} & 0 & s_{13} e^{-i \delta_{C P}} \\
0 & 1 & 0 \\
-s_{13} e^{i \delta_{C P}} & 0 & c_{13}
\end{array}\right) \\
& \times\left(\begin{array}{ccc}
c_{12} & s_{12} & 0 \\
-s_{12} & c_{12} & 0 \\
0 & 0 & 1
\end{array}\right)
\end{aligned}
$$

with $s_{i j}=\sin \theta_{i j}, c_{i j}=\cos \theta_{i j}$, and $\delta_{C P}$ being the $C P$ phase. We consider both cases for the orders of the neutrino masses, i.e., the normal hierarchy $\left(\left|m_{\nu}^{1}\right|<\left|m_{\nu}^{2}\right|<\left|m_{\nu}^{3}\right|\right)$ and the inverted hierarchy $\left(\left|m_{\nu}^{3}\right|<\left|m_{\nu}^{1}\right|<\left|m_{\nu}^{2}\right|\right)$.

The flavor structure of $M_{\nu}^{e}$ is the same as that of the Zee model. It has been known that the Zee model cannot explain the current neutrino oscillation data because of the too restricted structure of flavor violating couplings which only arise from the antisymmetric $3 \times 3 F$ matrix; see e.g., $[19,20] .{ }^{3}$ Although the flavor structure of $\mathcal{M}_{\nu}^{E}$ also takes a similar form as that of $\mathcal{M}_{\nu}^{e}$, another flavor violating source in the matrix $W$ is inserted into the mass matrix. Consequently, $\mathcal{M}_{\nu}^{E}$ has a different flavor mixing pattern. Hence, it can explain the current neutrino data. We note that such an additional flavor violating source vanishes if we take the masses of the extra leptons degenerate; i.e., $M_{1}=M_{2}=M_{3}$, by which $M_{k}^{2}$ and $G_{k}$ in Eq. (21) commute with $W^{\dagger}$ and then the effect of the $W$ matrix disappears by the unitarity. We also note that the contribution from $\mathcal{M}_{\nu}^{E}$ is typically much larger than that from $\mathcal{M}_{\nu}^{e}$, because the latter is proportional to the mass squared of the SM charged leptons. Therefore, the neutrino masses and the mixings are determined essentially only by the contribution from $\mathcal{M}_{\nu}^{E}$. We thus switch off the contribution from $\mathcal{M}_{\nu}^{e}$ hereafter for simplicity, which can be realized by taking $C_{e}=0$ or equivalently $\theta_{H}=0$.

\section{LEPTON FLAVOR VIOLATION}

In our model, new particles can contribute to LFV decays of the charged leptons. In this section, we discuss the constraints from $\ell \rightarrow \ell^{\prime} \gamma$ and $\ell \rightarrow \ell^{\prime} \ell^{\prime \prime} \ell^{\prime \prime \prime}$ types of LFV decays in the parameter sets which satisfy the current neutrino oscillation data.

Diagrams of the $\ell \rightarrow \ell^{\prime} \gamma$ processes are shown in Fig. 2. The branching ratios of these processes are calculated as

$\mathcal{B}\left(\ell_{i} \rightarrow \ell_{j} \gamma\right) \simeq \frac{48 \pi^{3} \alpha_{\mathrm{em}} C_{i j}}{G_{F}^{2}}\left[\left|\left(a_{R}\right)_{i j}\right|^{2}+\left|\left(a_{L}\right)_{i j}\right|^{2}\right]$,

where $\quad\left(\ell_{1}, \ell_{2}, \ell_{3}\right)=(e, \mu, \tau) \quad$ and $\quad\left(C_{21}, C_{31}, C_{32}\right)=$ $(1,0.1784,0.1736)$. In the above expression, the detailed formulas for $a_{L}$ and $a_{R}$ are presented in Appendix C. From the first diagram in Fig. 2, the structure of the $W$ matrix is

\footnotetext{
${ }^{3}$ If we take the general Yukawa interactions for leptons, then there is a corner of parameter space which can satisfy the current neutrino results [38-40].
} 

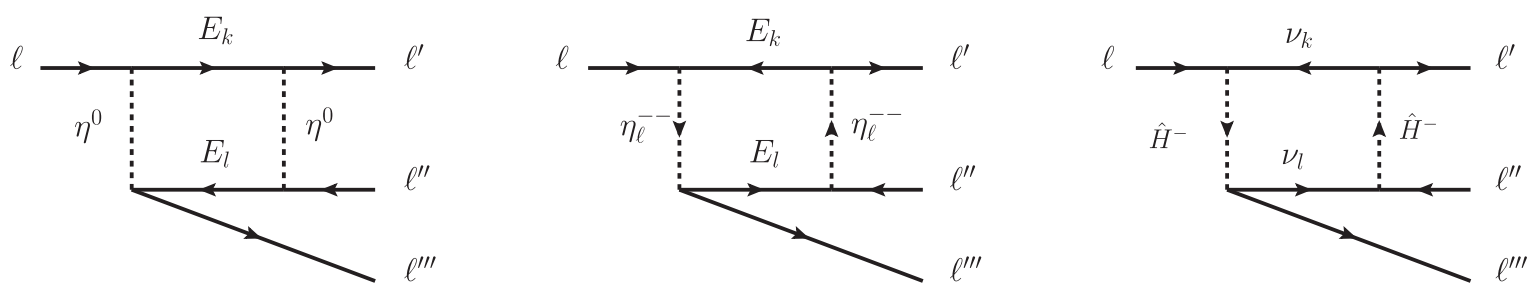

FIG. 3. Box diagrams for $\ell \rightarrow \ell^{\prime} \ell^{\prime \prime} \ell^{\prime \prime \prime}$ processes.

constrained. Since $W$ is a unitary matrix, we cannot simply take small values for each component. We can instead take the small mass difference among $E_{i}$ in order to suppress the contribution from the first diagram. On the other hand, the magnitude of the contributions from the second and the third diagrams can be easily suppressed by taking small values for the $F$ matrix elements. Typically, $\left|F_{i j}\right| \lesssim 10^{-3}$ is required from the constraint by the $\mu \rightarrow e \gamma$ data.

For the $\ell \rightarrow \ell^{\prime} \ell^{\prime \prime} \ell^{\prime \prime \prime}$ processes, the branching ratio of $\mu \rightarrow 3 e$ is most strongly constrained by the data among the six possible processes of this type. We thus concentrate on the constraint from the $\mu \rightarrow 3 e$ data. In this case, there are penguin type diagrams with the photon and the $Z$ boson exchanges and the box diagrams shown in Fig. 3. We confirm that the contribution from the box diagrams to the branching ratio is typically 8 orders of the magnitude smaller than the branching ratio of $\mu \rightarrow e \gamma$ in our scenario, so that we can safely neglect these contributions. In addition, it is usually the case that the contribution from the penguin diagram with the $Z$ boson exchange is negligibly smaller than that of the photon exchange [41]. ${ }^{4}$ Therefore, the dominant contribution arises from the penguin diagrams with the photon exchange which can be obtained by attaching the electron-positron line to the photon in the diagrams shown in Fig. 2. The branching ratio of $\mu \rightarrow 3 e$ is expressed as

$$
\begin{aligned}
\mathcal{B}(\mu \rightarrow e e \bar{e}) \simeq & \frac{6 \alpha_{\mathrm{em}}^{2}}{G_{F}^{2}}\left[\frac{2}{3}\left(\left|a_{L}\right|^{2}+\left|a_{R}\right|^{2}\right)\left(8 \ln \frac{m_{\mu}}{m_{e}}-11\right)\right. \\
& \left.+\left|b_{L}\right|^{2}+\left|b_{R}\right|^{2}-2\left(a_{R} b_{L}^{*}+a_{L} b_{R}^{*}+\text { c.c. }\right)\right],
\end{aligned}
$$

where the detailed formulas for $b_{L}$ and $b_{R}$ are presented in Appendix C. We note that the $\mu \rightarrow 3 e$ data typically do not further constrain the parameter region allowed by the $\ell \rightarrow \ell^{\prime} \gamma$ data.

Now, let us numerically show the prediction of the branching ratios of $\ell \rightarrow \ell^{\prime} \gamma$ and $\mu \rightarrow 3 e$ decays in the

\footnotetext{
${ }^{4}$ If there are new particles with large isospin charges which contribute to the effective $\bar{l} \ell^{\prime} Z$ vertex, then the $Z$ penguin diagram could be important as well as the photon one. This is, however, not the case in our model.
}

parameter sets which satisfy the current neutrino data. As aforementioned in Sec. III, we take $C_{e}=0$ (or $\theta_{H}=0$ ) in the numerical evaluation. We assume the $C P$ conservation in the Yukawa interaction terms. The $W$ matrix given in Eq. (19) becomes the orthogonal matrix which can be parametrized by the three angles entered in the $W$ matrix,

$$
\begin{aligned}
W= & \left(\begin{array}{ccc}
1 & 0 & 0 \\
0 & \cos w_{23} & -\sin w_{23} \\
0 & \sin w_{23} & \cos w_{23}
\end{array}\right)\left(\begin{array}{ccc}
\cos w_{13} & 0 & -\sin w_{13} \\
0 & 1 & 0 \\
\sin w_{13} & 0 & \cos w_{13}
\end{array}\right) \\
& \times\left(\begin{array}{ccc}
\cos w_{12} & -\sin w_{12} & 0 \\
\sin w_{12} & \cos w_{12} & 0 \\
0 & 0 & 1
\end{array}\right)
\end{aligned}
$$

We then take the following parameters as inputs:

$$
\begin{aligned}
& F_{12}, \quad F_{23}, \quad F_{13}, \quad w_{12}, \quad w_{23}, \quad w_{13}, \quad C_{E}, \\
& M_{1}, \quad M_{2}, \quad M_{3}, \quad m_{\eta^{ \pm}}, \quad m_{\hat{\eta}^{ \pm}}, V, \\
& \tan \beta, \quad m_{\eta_{\ell}^{ \pm \pm}}, \quad m_{\eta^{0}}, \quad m_{\hat{H}^{ \pm}} .
\end{aligned}
$$

The parameters in the first line are required for the neutrino mass calculation. For the calculation of the LFV decays, we also need to specify the parameters in the second line.

The current upper limits on the branching ratios of LFV decays of charged leptons are given at the $90 \%$ confidence level as

$$
\begin{aligned}
\mathcal{B}(\mu \rightarrow e \gamma) & <4.2 \times 10^{-13} \quad(\text { MEG }[42]), \\
\mathcal{B}(\tau \rightarrow e \gamma) & <3.3 \times 10^{-8} \quad(\text { BABAR }[43]), \\
\mathcal{B}(\tau \rightarrow \mu \gamma) & <4.4 \times 10^{-8} \quad(\text { BABAR }[43]), \\
\mathcal{B}(\mu \rightarrow e e e) & <1.0 \times 10^{-12} \quad(\text { SHINDRUM [44]). }
\end{aligned}
$$

We refer to the neutrino oscillation data given in Ref. [45], and we apply the $3 \sigma$ allowed ranges of two squared mass differences and three mixing angles to our analysis.

In Fig. 4, we show various correlations between the branching ratios of the LFV decays. In these plots, we scan six parameters $F_{i j}$ and $w_{i j}$, and fix other parameters as written in the caption of Fig. 4. The coefficient $C_{E}$ is determined so as to reproduce the mass squared difference of atmospheric neutrinos, i.e., $\Delta m_{\mathrm{atm}}^{2}=\left|\left(m_{\nu}^{3}\right)^{2}-\left(m_{\nu}^{1}\right)^{2}\right|$. 

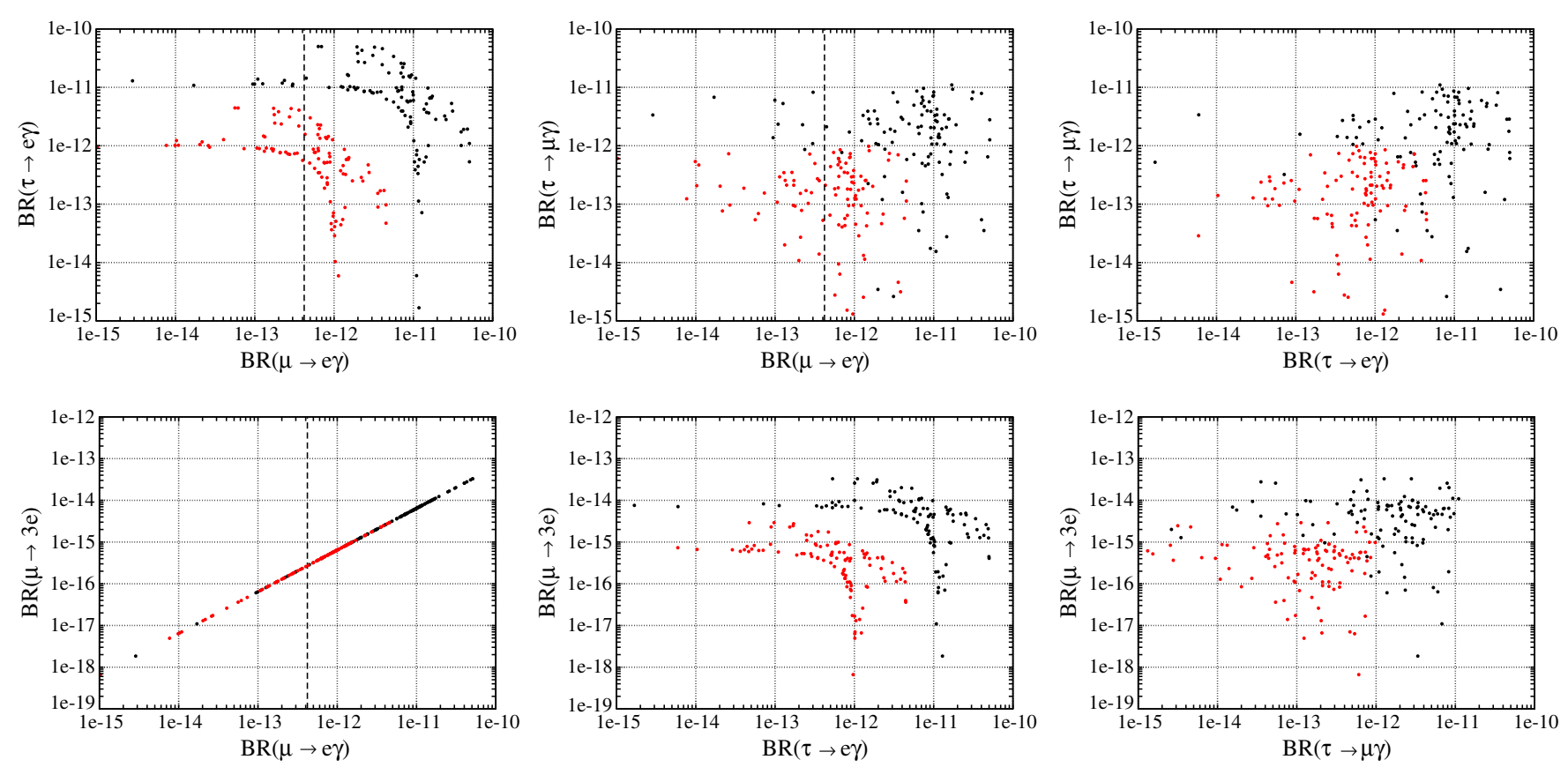

FIG. 4. Correlations between BRs of $\ell \rightarrow \ell^{\prime} \gamma$ (upper figures) and those of $\ell \rightarrow \ell^{\prime} \gamma$ and $\mu \rightarrow 3 e$ (lower figures) in the case of $\tan \beta=30$ (100) for black (red) points. The dashed vertical line shows the current upper limit on the branching ratio of $\mu \rightarrow e \gamma$. In these plots, we fix $V=10 \mathrm{TeV}, \quad\left(M_{1}, M_{2}, M_{3}\right)=(300,301,302) \mathrm{GeV}, \quad m_{\eta^{ \pm}}=450 \mathrm{GeV}, \quad m_{\hat{\eta}^{ \pm}}=m_{\eta_{f}^{ \pm \pm}}=m_{\hat{H}^{ \pm}}=400 \mathrm{GeV}, \quad$ and $m_{\eta^{0}}=63 \mathrm{GeV}$. We scan the six parameters of $F_{i j}$ and $w_{i j}$ with the ranges of $-10^{-3} \leq F_{i j} \leq 10^{-3}$ and $-\pi / 2 \leq w_{i j} \leq \pi / 2$. All points satisfy the neutrino oscillation data assuming the normal hierarchy case for the neutrino masses.

All these points satisfy the current neutrino oscillation data assuming the normal hierarchy for neutrino masses, where the black (red) points show the case with $\tan \beta=30(100) .^{5}$ We see that the red points are given in the lower-left region of these planes as compared with the black points, because values of the branching ratios of $\ell \rightarrow \ell^{\prime} \gamma$ are dominantly determined by the second term of Eq. (C2), which is proportional to $c_{\beta}$. We note that the loop contributions of $\eta_{\ell}^{ \pm \pm}$and $\hat{H}^{ \pm}$are unimportant as long as $\left|F_{i j}\right|$ becomes larger, whose magnitude is typically taken to be smaller than $\mathcal{O}\left(10^{-3}\right)$. We see that the $\mu \rightarrow$ er data give the most severe constraint on the parameter space, because of its strongest upper bound on the branching ratio. For the other two modes $\tau \rightarrow \mu \gamma$ and $\tau \rightarrow e \gamma$, our predictions are typically smaller than the current limit by 2 or more orders of magnitude, because the branching ratio has already been highly suppressed by the $\mu \rightarrow e \gamma$ data. It is also seen that the branching ratio of $\mu \rightarrow 3 e$ is significantly lower than the current upper limit. We observe an anticorrelation between the branching ratios of $\mu \rightarrow e \gamma$ and $\tau \rightarrow e \gamma$, see the most

\footnotetext{
${ }^{5}$ We confirm that all the relevant parameters in the Lagrangian are small enough in order to ensure the perturbativity. In fact, the typical magnitudes of the elements of new Yukawa matrices $y_{E}$ and $F$ are given by $\mathcal{O}\left(10^{-2}\right)$ and $\mathcal{O}\left(10^{-3}\right)$, respectively. In addition, that of the coefficient $C_{E}$ is found to be $\mathcal{O}\left(10^{-4}\right)$. This can be realized by taking $s_{\theta_{\eta}}=\mathcal{O}\left(10^{-2}\right)$, or equivalently taking $\xi_{2}=\mathcal{O}\left(10^{-2}\right)$.
}

upper-left panel, which is predicted by our characteristic flavor structure of the Yukawa interactions. In addition, we find a very strong correlation between the branching ratios of $\mu \rightarrow e \gamma$ and $\mu \rightarrow 3 e$. This can be understood from the fact that the $\left|a_{L}\right|^{2}$ and $\left|a_{R}\right|^{2}$ terms given in Eq. (27) mainly determine the size of the branching ratio of $\mu \rightarrow 3 e$.

Similar plots but for the inverted hierarchy case are shown in Fig. 5. We see that $\mathcal{B}(\tau \rightarrow \mu \gamma)$ tends to have similar values with the order of $10^{-11}\left(10^{-12}\right)$ for $\tan \beta=$ $30(100)$ as a function of the other branching ratios, which cannot be seen in the normal hierarchy case. The other behavior is quite similar to the normal hierarchy case.

Let us give a comment on cases for the other sets of the fixed parameters in the above analysis. Among the fixed parameters, the mass differences between the extra leptons can significantly affect the results of the LFV branching ratios. For larger values of the mass difference, these branching ratios tend to become larger, because the suppression by the unitarity of the $W$ matrix becomes weaker. Therefore, larger values of $\tan \beta$ or $V$ are required to avoid the constraint from the $\mu \rightarrow e \gamma$ data. Varying the other mass parameters such as $m_{\eta^{ \pm}}$does not change the above results significantly.

\section{PHENOMENOLOGY}

In this section, we discuss phenomenological consequences of our model. 

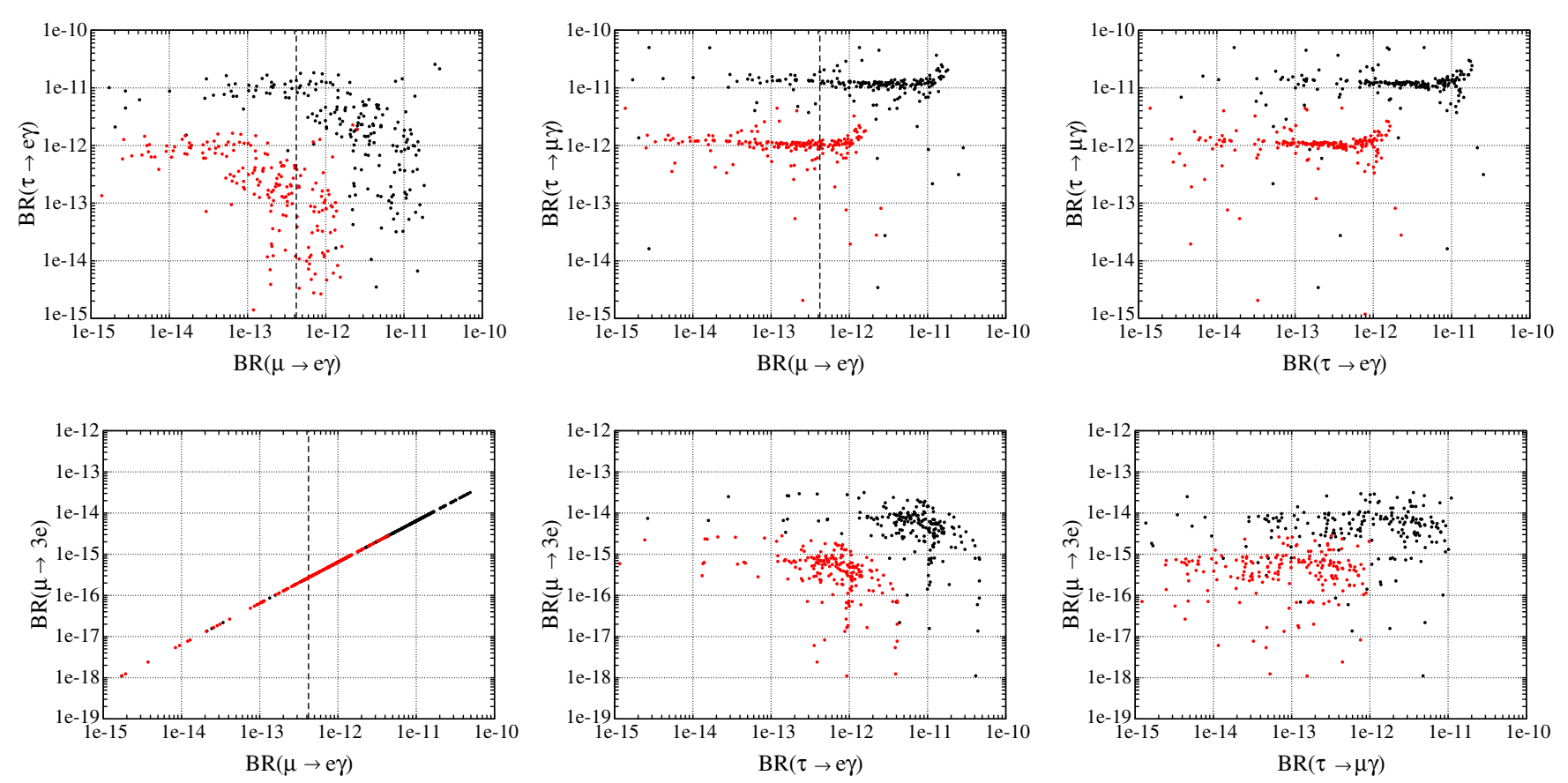

FIG. 5. Same as Fig. 4, but for the inverted hierarchy case for the neutrino masses.

\section{A. Dark matter physics}

As we discussed in Sec. II, the lightest $Z_{2}^{\text {rem }}$-odd particle can be a candidate of dark matter, i.e., the complex scalar $\eta^{0}$ or the complex gauge boson $Y^{\mu}$. A scenario with the gaugeboson dark matter $Y^{\mu}$ is similar to that discussed in Ref. [16], and it has been shown that the observed relic abundance is difficult to be explained, due to the too large mass of $Y^{\mu}(\gtrsim 8 \mathrm{TeV})$. We thus consider the scalar boson $\eta^{0}$ as the dark matter candidate hereafter.

The scalar boson $\eta^{0}$ has trilinear interaction terms with neutral $Z_{2}^{\text {rem }}$ - and $C P$-even scalar bosons $H_{i}(i=1,2,3)$, among which $H_{1}$ can be identified with the discovered Higgs boson $(h)$ with the mass of $125 \mathrm{GeV}$. Therefore, the phenomenology of dark matter is similar to the Higgs portal scenario in which annihilation processes occur via the $s$-channel Higgs-boson mediations.

If the dark matter mass $m_{\eta^{0}}$ is smaller than $2 m_{W}$ and if the additional Higgs bosons $\mathrm{H}_{2}$ and $\mathrm{H}_{3}$ are much heavier than $2 m_{W}$, the dominant annihilation process is $\eta^{0} \eta^{0 *} \rightarrow$ $h^{(*)} \rightarrow f \bar{f}$ with $f \neq t$, whose thermal averaged cross section is evaluated at the leading order as

$$
\langle\sigma v\rangle \simeq \sum_{f} \frac{m_{f}}{\pi v}\left(1-\frac{4 m_{f}^{2}}{m_{\eta^{0}}^{2}}\right)^{3 / 2} \frac{\lambda_{h \eta \eta}^{2}}{\left(4 m_{\eta^{0}}^{2}-m_{h}^{2}\right)^{2}+m_{h}^{2} \Gamma_{h}^{2}},
$$

where $\Gamma_{h}$ is the width of $h(\sim 4 \mathrm{MeV})$ and $\lambda_{h \eta \eta}$ is the dimensionful $\eta^{0} \eta^{0 *} h$ coupling. From Eq. (31), the annihilation cross section becomes significant when $m_{\eta^{0}}$ is getting close to $m_{h} / 2$ due to the resonant effect of $h$, so that smaller values of $\lambda_{h \eta \eta}$ are required to keep the observed relic abundance of dark matter, $\Omega_{\mathrm{DM}} h^{2} \simeq 0.12$ [46].

On the other hand, constraints from dark matter direct detections have to be taken into account. In our scenario, the dark matter scattering with a nucleon $N$ through the $t$-channel Higgs mediation becomes most important. Using the effective vertex, which is given by

$$
\mathcal{L}_{N}=g_{N} \bar{N} N h,
$$

with $g_{N} \simeq 1.1 \times 10^{-3}$ [47], the scattering cross section is expressed as

$$
\sigma_{N} \simeq \frac{g_{N}^{2} \lambda_{h \eta \eta}^{2}}{4 \pi\left(m_{N}+m_{\eta^{0}}\right)^{2}} \frac{m_{N}^{2}}{m_{h}^{4}}
$$

where $m_{N}$ is the mass of the nucleon. We here neglect the 3-momentum of the dark matter.

In Fig. 6, we show the combined results for the calculations of the relic abundance and the bound from the direct search experiment (XENON1T) [48]. The red curve represents the upper limit on the normalized $h \eta^{0} \eta^{0 *}$ coupling by the $\operatorname{VEV} \lambda_{h \eta \eta} / v$ as a function of the dark matter mass $m_{\eta^{0}}$. The required value of $\lambda_{h \eta \eta} / v$ to satisfy $\Omega_{\mathrm{DM}} h^{2}=$ 0.12 is shown as the black curve. As already mentioned, smaller values of $\lambda_{h \eta \eta}$ are required to keep the observed value of the abundance when the dark matter mass is around the resonance region $\sim m_{h} / 2$. Our dark matter candidate can simultaneously satisfy both the relic abundance and the direct detection bounds at $m_{\eta^{0}} \sim m_{h} / 2$ as it 


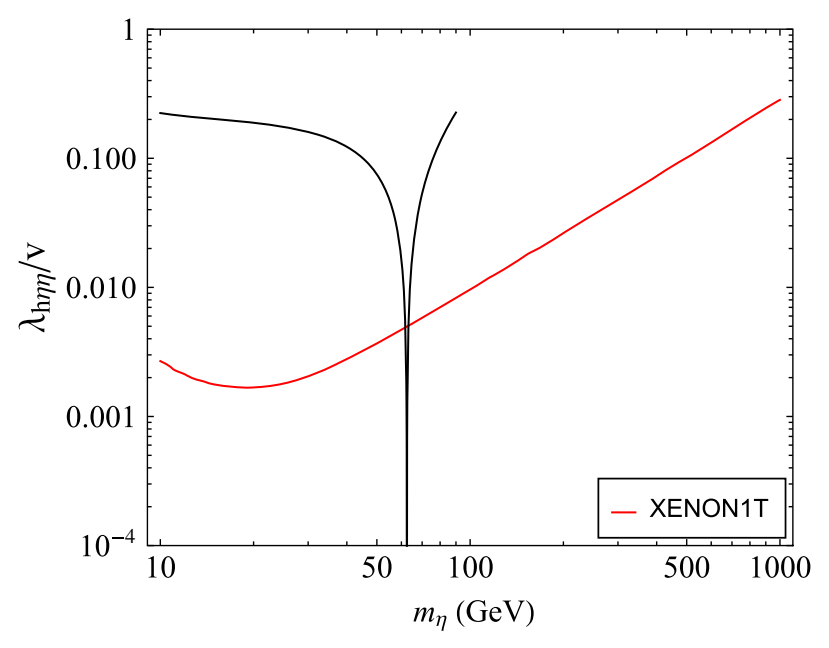

FIG. 6. Combined results for the dark matter relic abundance and the constraint from the dark matter direct search experiment. The red curve shows the upper limit on the normalized coupling by the $\operatorname{VEV} \lambda_{h \eta \eta} / v$ as a function of the dark matter mass given by the XENON1T experiment. The black curve shows the required value of $\lambda_{h \eta \eta} / v$ satisfying $\Omega_{\mathrm{DM}} h^{2}=0.12$ as a function of the dark matter mass. has been known in Higgs portal models; see e.g., [49-51]. Another scenario with a much larger mass ( $\gtrsim$ a few $\mathrm{TeV}$ ) may also be considered for the dark matter to satisfy both the dark matter data. However, we do not discuss details of this case because such a scenario strongly depends on the parameters of extra fields. Instead, we only have shown that there is at least a solution in our model to satisfy the dark matter data in addition to the neutrino data.

\section{B. Collider physics}

In our model, there are many new particles, which can potentially be produced at collider experiments. However, in the case with $V \gg v$, our model effectively coincides with the THDM with a special Yukawa interaction, which gives extra bosons $H^{ \pm}, A$, and $H_{2}$. Because of the special Yukawa interaction, they can decay into quarks with different flavors such as $A / H_{2} \rightarrow t c$ and $H^{ \pm} \rightarrow t s$ [37]. Dedicated simulation studies for these flavor violating decays of the extra Higgs bosons at the LHC have been performed in Ref. [52]. In addition, there are other extra particles whose masses are proportional to $V$ such as $\eta_{\ell}^{ \pm \pm}$, $\eta^{ \pm}, \hat{\eta}^{ \pm}, \eta^{0} \hat{H}^{ \pm}$, and $H_{3}$. They can also be detected at the LHC if the associated coupling constants are small enough.

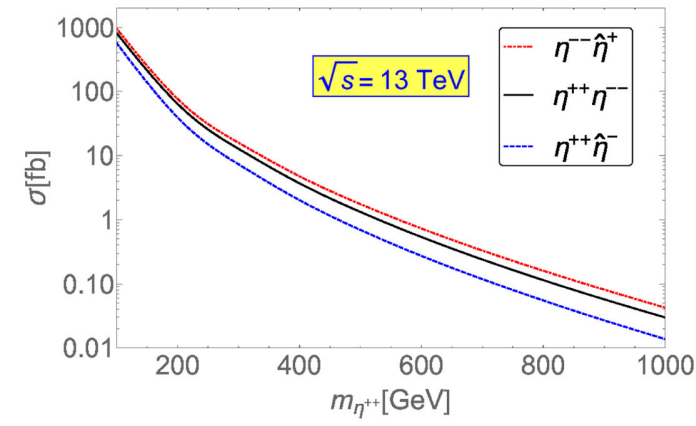

FIG. 7. Production cross sections for the $p p \rightarrow \gamma^{*} / Z^{*} \rightarrow \eta_{\ell}^{++} \eta_{\ell}^{--}$and $p p \rightarrow W^{ \pm *} \rightarrow \eta_{\ell}^{ \pm \pm} \hat{\eta}^{\mp}$ processes as a function of $m_{\eta_{\ell}^{ \pm \pm}}$. The collision energy is taken to be $13 \mathrm{TeV}$ (left) and $27 \mathrm{TeV}$ (right). For the $\eta_{\ell}^{ \pm \pm} \hat{\eta}^{\mp}$ productions, we take $m_{\hat{\eta}^{ \pm}}=m_{\eta_{\ell}^{ \pm \pm}}$.
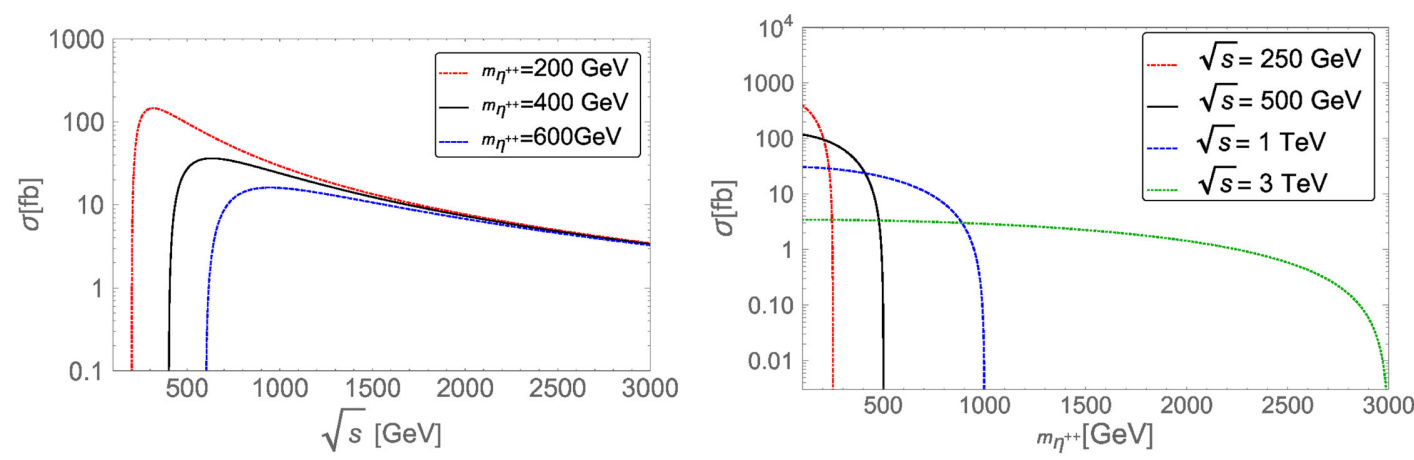

FIG. 8. Production cross sections for the $e^{+} e^{-} \rightarrow \gamma^{*} / Z^{*} \rightarrow \eta_{\ell}^{++} \eta_{\ell}^{--}$process at future lepton colliders. The left panel shows the dependence on the center of mass energy $\sqrt{s}$ with fixed values of the mass of $\eta_{\ell}^{ \pm \pm}$to be 200, 400, and $600 \mathrm{GeV}$. The right panel shows the dependence on the mass of $\eta_{\ell}^{ \pm \pm}$with fixed values of $\sqrt{s}$ to be $255 \mathrm{GeV}, 500 \mathrm{GeV}, 1 \mathrm{TeV}$, and $3 \mathrm{TeV}$. 
TABLE III. Benchmark inputs and corresponding outputs, where $\mathcal{B}_{i j}$ denote $\mathcal{B}\left(\eta_{\ell^{ \pm \pm}} \rightarrow \ell_{i}^{ \pm} \ell_{j}^{ \pm} \eta^{0}\right)$, and $\Gamma_{\text {tot }}$ is the total width of $\eta_{\ell^{ \pm \pm}}$. In this table, $w_{i j}, \mathcal{B}_{i j}$, and $\Gamma_{\text {tot }}$ are given in the units of rad, $\%$, and $\mathrm{keV}$, respectively. The other input parameters are fixed to be $V=10 \mathrm{TeV},\left(M_{1}, M_{2}, M_{3}\right)=(300,301,302) \mathrm{GeV}, m_{\eta^{ \pm}}=450 \mathrm{GeV}, m_{\hat{\eta}^{ \pm}}=m_{\eta_{t}^{ \pm \pm}}=m_{\hat{H}^{ \pm}}=400 \mathrm{GeV}$, and $m_{\eta^{0}}=63 \mathrm{GeV}$. All these benchmark points satisfy the neutrino oscillation data and the LFV data assuming the normal hierarchy case for the neutrino masses.

\begin{tabular}{|c|c|c|c|c|c|c|c|c|c|c|c|c|}
\hline \multicolumn{6}{|c|}{ Inputs } & \multicolumn{7}{|c|}{ Outputs } \\
\hline$F_{12} \times 10^{4}$ & $F_{23} \times 10^{4}$ & $F_{13} \times 10^{4}$ & $w_{12}$ & $w_{23}$ & $w_{13}$ & $\mathcal{B}_{e e}$ & $\mathcal{B}_{\mu \mu}$ & $\mathcal{B}_{\tau \tau}$ & $\mathcal{B}_{e \mu}$ & $\mathcal{B}_{\mu \tau}$ & $\mathcal{B}_{e \tau}$ & $\Gamma_{\text {tot }}$ \\
\hline-3.03 & 8.13 & 8.44 & -1.52 & -0.184 & 0.573 & 12.3 & 1.2 & 5.5 & 15.7 & 34.3 & 31.0 & 8.48 \\
\hline-1.36 & -3.99 & -4.22 & -0.988 & -1.37 & 0.0325 & 0. & 46.9 & 1.6 & 8.7 & 42.4 & 0.4 & 2.08 \\
\hline 0.324 & -6.91 & 5.46 & 0.677 & -1.39 & -0.0841 & 0.1 & 27.8 & 1.7 & 19.1 & 50.3 & 1.0 & 4.78 \\
\hline 0.0334 & 5.19 & -4.65 & 1.56 & 0.149 & 0.958 & 35.8 & 0.2 & 7.5 & 16.0 & 7.7 & 32.9 & 2.99 \\
\hline 2.11 & 4.35 & 4.09 & -1.05 & -1.47 & 0.00966 & 0. & 46.6 & 0.5 & 9.8 & 42.5 & 0.5 & 2.20 \\
\hline
\end{tabular}

One of the most interesting signatures in our model arises from the doubly charged scalar bosons $\eta_{\ell}^{ \pm \pm}$. At the LHC, they can be created in pairs via the Drell-Yan process $p p \rightarrow \gamma^{*} / Z^{*} \rightarrow \eta_{\ell}^{++} \eta_{\ell}^{--}$and in association with the singly charged scalar bosons $p p \rightarrow W^{ \pm *} \rightarrow \eta_{\ell}^{ \pm \pm} \hat{\eta}^{\mp} / \eta_{\ell}^{ \pm \pm} \eta^{\mp}$ [53]. In Fig. 7, we show the cross sections for these production processes at the $p p$ colliders with the collision energy of $13 \mathrm{TeV}$ (left) and $27 \mathrm{TeV}$ (right). We use NNPDF2.3-LO [54] for the parton distribution functions. We here neglect effects of the mixing angle $\theta_{\eta}$ on the associated production cross section, because by the analyses for neutrino masses in Sec. IV we have typically $\theta_{\eta}=\mathcal{O}\left(10^{-2}\right)$ which is sufficiently small. It can be seen that the cross section for the pair production with $m_{\eta_{\ell}^{ \pm+}} \simeq 400 \mathrm{GeV}$ can be a few (a few tens of) fb at $\sqrt{s}=13 \mathrm{TeV}(27 \mathrm{TeV})$. Slightly larger (smaller) values are obtained for the cross section of the associated production $\eta_{\ell}^{++} \hat{\eta}^{-}\left(\eta_{\ell}^{--} \hat{\eta}^{+}\right)$. In Fig. 8, we also show the pair production cross section at future lepton colliders, $e^{+} e^{-} \rightarrow \gamma^{*} / Z^{*} \rightarrow \eta_{\ell}^{++} \eta_{\ell}^{--}$, as a function of the center of mass energy $\sqrt{s}$ (left) and the mass $m_{\eta_{t}^{ \pm \pm}}$(right).

Using the mass spectrum assumed in the analyses for the neutrino masses and LFV decays, the decay pattern of $\eta_{\ell}^{ \pm \pm}$ is determined to be $\eta_{\ell}^{ \pm \pm} \rightarrow E_{k}^{ \pm} \ell_{i}^{ \pm} \rightarrow \ell_{i}^{ \pm} \ell_{j}^{ \pm} \eta^{0}$, where $\eta^{0}$ is the dark matter candidate. The intermediate state $E_{k}^{ \pm}$can be on shell in this case. The flavor of the same-sign dilepton in the final state is determined by the $F$ and $W$ matrices, which are constrained by the neutrino oscillation data and the LFV data. Therefore, future measurements of the flavor of the same-sign dilepton system can give constraints on the structure of the Yukawa interaction in our model, and it might be able to provide a hint for the mechanism for the neutrino mass generation.

The partial decay rates of $E_{i}^{ \pm}$and $\eta_{\ell}^{ \pm \pm}$are calculated as

$\Gamma\left(E_{i}^{ \pm} \rightarrow \ell_{j}^{ \pm} \eta^{0}\right)=\frac{M_{E}}{32 \pi}\left(1-\frac{m_{\eta^{0}}^{2}}{M_{E}^{2}}\right)^{2}\left[\left|\left(h_{R}\right)_{i j}\right|^{2}+\left|\left(h_{L}\right)_{i j}\right|^{2}\right]$,

$$
\begin{aligned}
\Gamma\left(\eta_{\ell^{ \pm \pm}} \rightarrow \ell_{i}^{ \pm} \ell_{j}^{ \pm} \eta^{0}\right)= & \frac{m_{\eta_{\ell}^{ \pm \pm}}}{16 \pi} \frac{M_{E}}{32 \pi \Gamma_{E}}\left(1-\frac{m_{\eta_{\ell}^{ \pm \pm}}^{2}}{M_{E}^{2}}\right)^{2}\left(1-\frac{m_{\eta^{0}}^{2}}{M_{E}^{2}}\right)^{2} \\
& \times\left[\left|\left(2 W^{T} F h_{R}\right)_{i j}\right|^{2}+\left|\left(2 W^{T} F h_{L}\right)_{i j}\right|^{2}\right],
\end{aligned}
$$

where $h_{L}$ and $h_{R}$ are given in Eq. (C10), and the mass of the SM charged leptons is neglected. In these expressions, small differences of the masses and the widths of $E_{i}$ are ignored ${ }^{6}$; i.e., $M_{E} \equiv M_{1}\left(=M_{2}=M_{3}\right)$ and $\Gamma_{E} \equiv$ $\Gamma_{E_{1}}\left(=\Gamma_{E_{2}}=\Gamma_{E_{3}}\right)$. The total width $\Gamma_{E}$ is typically of order $0.1 \mathrm{GeV}$, so that the narrow width approximation is valid for the calculation of the decay rate of $\eta_{\ell}^{ \pm \pm}$.

In Tables III and IV, we give several benchmark points which satisfy the neutrino data and the LFV data for the cases of the normal and the inverted hierarchies, respectively. The other input parameters are fixed to be $V=10 \mathrm{TeV}, \quad\left(M_{1}, M_{2}, M_{3}\right)=(300,301,302) \mathrm{GeV}$, $m_{\eta^{ \pm}}=450 \mathrm{GeV}$, and $m_{\hat{\eta}^{ \pm}}=m_{\eta_{\ell}^{ \pm \pm}}=m_{\hat{H}^{ \pm}}=400 \mathrm{GeV}$. The dark matter mass $m_{\eta^{0}}$ is fixed to be $63 \mathrm{GeV}$ in order to satisfy the relic abundance and the constraint from the direct search experiment, see Sec. VA. For each point, we show our predictions for the branching ratios of $\eta_{\ell}^{ \pm \pm}$and its total width $\Gamma_{\text {tot }}$. It is seen that the width of $\eta_{\ell}^{ \pm \pm}$is typically given to be of the order of $\mathrm{keV}$, because the couplings $F_{i j}$ are taken to be $\mathcal{O}\left(10^{-3}-10^{-4}\right)$ in order to avoid the constraint from LFV decays of charged leptons. Depending on these benchmark points, $\eta_{\ell}^{ \pm \pm}$can predominantly decay into the same-sign dilepton with various combinations of their flavors. Because the decay of $\eta_{\ell}^{ \pm \pm}$ contains missing energies which are carried by the dark matter $\eta^{0}$, the invariant mass distribution of the same-sign dilepton system does not have a peak at around the mass of $\eta_{\ell}^{ \pm \pm}$. This property is different from that of doubly charged Higgs bosons from an $S U(2)_{L}$ triplet or a singlet field,

\footnotetext{
${ }^{6}$ The small mass difference is required in order to reproduce the neutrino mixing data and to avoid the constraints from LFV decays of charged leptons.
} 
TABLE IV. Same as Table III, but for the inverted hierarchy case.

\begin{tabular}{|c|c|c|c|c|c|c|c|c|c|c|c|c|}
\hline \multicolumn{6}{|c|}{ Inputs } & \multicolumn{7}{|c|}{ Outputs } \\
\hline$F_{12} \times 10^{4}$ & $F_{23} \times 10^{4}$ & $F_{13} \times 10^{4}$ & $w_{12}$ & $w_{23}$ & $w_{13}$ & $\overline{\mathcal{B}_{e e}}$ & $\mathcal{B}_{\mu \mu}$ & $\mathcal{B}_{\tau \tau}$ & $\mathcal{B}_{e \mu}$ & $\mathcal{B}_{\mu \tau}$ & $\mathcal{B}_{e \tau}$ & $\overline{\Gamma_{\text {tot }}}$ \\
\hline 2.41 & -0.716 & 4.34 & 1.43 & 0.957 & 0.0947 & 0. & 65.9 & 0 . & 1.1 & 33 & 0.1 & 1.19 \\
\hline 6.81 & -0.982 & -6.92 & -1.08 & -0.427 & 1.08 & 1.4 & 1.0 & 13.3 & 21.0 & 7.8 & 55.4 & 3.02 \\
\hline 2.61 & -0.767 & 6.64 & -0.197 & -1.41 & 0.0189 & 0. & 3.0 & 0. & 92.7 & 1.8 & 2.4 & 2.76 \\
\hline-7.02 & 1.308 & -5.32 & 1.36 & 0.733 & 0.205 & 0. & 42.8 & 0.1 & 4.2 & 52.9 & 0.1 & 1.85 \\
\hline-6.33 & -0.792 & 4.32 & -0.823 & -0.129 & 1.33 & 7.2 & 0. & 5.2 & 0.1 & 0.1 & 87.3 & 1.19 \\
\hline
\end{tabular}

which can decay into the same-sign dilepton without missing energy. Therefore, the current bounds on the mass of such doubly charged Higgs bosons, around $800 \mathrm{GeV}$ depending on the flavor of the final state leptons at the LHC [55], cannot be applied to that on $\eta_{\ell}^{ \pm \pm}$. In order to extract the bound on the mass of $\eta_{\ell}^{ \pm \pm}$from current experiments and its discovery potential at future experiments, dedicated simulation studies are needed, which are beyond the scope of this paper.

For the sake of completeness, let us discuss the phenomenology for the singly charged scalar bosons $\eta^{ \pm}, \hat{\eta}^{ \pm}$, and $\hat{H}^{ \pm}$. For simplicity, we neglect the effect of the small mixing angle $\theta_{\eta}\left(\theta_{H}\right)$ between $\eta^{ \pm}$and $\hat{\eta}^{ \pm}\left(H^{ \pm}\right.$and $\left.\hat{H}^{ \pm}\right)$. These bosons can be produced in pair via the Drell-Yan process at collider experiments. In addition, $\hat{\eta}^{ \pm}$can also be produced in association with $\eta_{\ell}^{ \pm \pm}$as already discussed in the above text; see also Fig. 7 for its production cross section. Their decay processes can be $\eta^{ \pm} / \hat{\eta}^{ \pm} \rightarrow \nu_{i} E_{k}^{ \pm} \rightarrow$ $\nu_{i} \ell_{j}^{ \pm} \eta^{07}$ and $\hat{H}^{ \pm} \rightarrow \ell_{i}^{ \pm} \nu_{j}$. Because of the $Z_{2}^{\text {rem }}$ symmetry, the decays of $\eta^{ \pm}$and $\hat{\eta}^{ \pm}$include the dark matter. The decay of $\eta^{ \pm}\left(\hat{\eta}^{ \pm}\right.$and $\left.\hat{H}^{ \pm}\right)$occurs via the Yukawa coupling $y_{E}(F)$, so that the flavor dependence of the charged lepton in the final state could be different among the decaying particles. We note that the singly charged scalar bosons in the inert doublet model can decay into the $W$ boson and a lighter $Z_{2}$-odd scalar particle. Therefore, the signatures from the decays of $\eta^{ \pm}$and $\hat{\eta}^{ \pm}$can be different from those in the inert doublet model $[56,57]$. On the other hand, the decay property of $\hat{H}^{ \pm}$is quite similar to that of singly charged scalar bosons in the Zee model [58-60].

\section{CONCLUSIONS}

We have proposed a new model for the generation of tiny neutrino masses based on the 3-3-1 gauge symmetry within the minimal fermion content required for the gauge anomaly cancellation. In this model, the source for lepton number violation is obtained by extending the minimal Higgs sector of 3-3-1 models with an additional $S U(3)_{L}$ triplet scalar field. Majorana masses for the active neutrinos

\footnotetext{
${ }^{7}$ If $\eta_{\ell}^{ \pm \pm}$are lighter than $\hat{\eta}^{ \pm}$, the $\hat{\eta}^{ \pm} \rightarrow \eta_{\ell}^{ \pm \pm} W^{\mp(*)}$ processes are also possible.
}

are generated at one-loop level. We have found the parameter sets which satisfy the current neutrino data under the constraint from the LFV decays of the charged leptons such as $\mu \rightarrow e \gamma$ and $\mu \rightarrow 3 e$.

In our model, $Z_{2}^{\text {rem }}$ appears as a remnant symmetry after the breaking of the electroweak symmetry and that of the global $U(1)^{\prime}$ symmetry, where the latter symmetry is introduced to avoid the dangerous flavor changing neutral current. The symmetry $Z_{2}^{\text {rem }}$ guarantees the stability of the dark matter candidate which is the lightest neutral $Z_{2}^{\text {rem }}$-odd scalar particle $\eta^{0}$. We have confirmed that the dark matter candidate can satisfy the relic abundance and the direct search results when the dark matter mass is taken to be at around half of the discovered Higgs boson mass.

We then discussed the collider phenomenology of our model. One of the most interesting signatures arises from productions and decays of the doubly charged scalar bosons $\eta_{\ell}^{ \pm \pm}$, because of the characteristic flavor dependence of the same-sign dilepton in the final state. Even if $\eta_{\ell}^{ \pm \pm}$ are too heavy to be detected at collider experiments, the Higgs sector of our model, which effectively coincides with the THDM at $V \gg v$, predicts the special structure of the Yukawa interaction to the SM quarks. The extra Higgs bosons can then mainly decay into quarks with different flavors, so that the detection of such bosons would be important to test our model.

In conclusion, our model can give an interesting testable example of the 3-3-1 scenario, where the number of generation of quarks and leptons, neutrino oscillation, and dark matter can be explained simultaneously.

\section{ACKNOWLEDGMENTS}

The authors would like to thank Niko Koivunen and Katri Huitu for useful discussions. The works of A. D. and S. K. were supported in part by JSPS, Grant-in-Aid for Scientific Research, No. 18F18321. K. E. was supported in part by the Sasakawa Scientific Research Grant from The Japan Science Society. S. K. was supported in part by Grant-in-Aid for Scientific Research on Innovative Areas, the Ministry of Education, Culture, Sports, Science and Technology, No. 16H06492 and No. 18H04587, and also by JSPS, Grant-in-Aid for Scientific Research, Grant No. 18F18022. The work of K. Y. was supported in part 
by the Grant-in-Aid for Early-Career Scientists, No. 19K14714.

\section{APPENDIX A: STATIONARY CONDITIONS}

We show that the vanishment of the VEV $v^{\prime}$ given in Eq. (5) is guaranteed due to the remnant $\tilde{Z}_{2}$ symmetry which is discussed in Sec. II. The stationary conditions for each neutral component of the scalar field can be expressed as

$$
\left.\frac{\partial V}{\partial \varphi^{0}}\right|_{0}=0
$$

where $\left.\right|_{0}$ represents taking all the fields to be zero after the derivative. We note that nonzero values of the left-hand side appear for the real component of the scalar field due to the assumption of $C P$ conservation in the Higgs potential. For the condition of the real component of $\eta_{1}^{0}$, we obtain

$$
m_{13}^{2} V+\frac{v^{\prime} v}{2}\left(\sqrt{2} \mu s_{\beta}+V \rho_{13} c_{\beta}\right)=0 .
$$

Because the $\tilde{Z}_{2}$ symmetry in the Lagrangian forbids the $m_{13}^{2}$ term, the above equation is satisfied by taking $v^{\prime}=0$ with $v \neq 0$ for arbitrary nonzero values of the term inside the parentheses. In this case, the condition for the real component of $\eta_{3}^{0}$ is simultaneously satisfied, and the nontrivial conditions appear from those for the real components of $\phi_{a}^{0}(a=1,2,3)$ as follows:

$v\left[2 m_{1}^{2} c_{\beta}+v^{2} c_{\beta}\left(2 \lambda_{1} c_{\beta}^{2}+\lambda_{12} s_{\beta}^{2}\right)+V^{2} \lambda_{13} c_{\beta}-\sqrt{2} V \mu s_{\beta}\right]=0$,

$v\left[2 m_{2}^{2} s_{\beta}+v^{2} s_{\beta}\left(2 \lambda_{2} s_{\beta}^{2}+\lambda_{12} c_{\beta}^{2}\right)+V^{2} \lambda_{23} s_{\beta}-\sqrt{2} V \mu c_{\beta}\right]=0$,

$V\left[2 m_{3}^{2}+2 V^{2} \lambda_{3}+v^{2}\left(\lambda_{13} c_{\beta}^{2}+\lambda_{23} s_{\beta}^{2}\right)\right]-\sqrt{2} \mu v^{2} s_{\beta} c_{\beta}=0$.

We can solve these equations in terms of $m_{1}^{2}, m_{2}^{2}$, and $m_{3}^{2}$.

\section{APPENDIX B: MASS FORMULAS FOR THE SCALAR BOSONS}

We first give the mass formulas for the $Z_{2}^{\text {rem }}$-even scalar bosons. The neutral components of the scalar triplet fields can be expressed as

$$
\phi_{a}^{0}=\frac{1}{\sqrt{2}}\left(\phi_{a}^{R}+v_{a}+i \phi_{a}^{I}\right), \quad(a=1,2,3),
$$

with $v_{3}=V$. There are three pairs of singly charged, three $C P$-odd, and three $C P$-even scalar states in the $Z_{2}^{\text {rem }}$-even sector. Their mass eigenstates are obtained by introducing the following orthogonal transformations:

$$
\begin{aligned}
& \left(\begin{array}{c}
\phi_{1}^{ \pm} \\
\phi_{2}^{ \pm} \\
\phi_{\ell}^{ \pm}
\end{array}\right)=\left(\begin{array}{ccc}
c_{\beta} & s_{\beta} & 0 \\
-s_{\beta} & c_{\beta} & 0 \\
0 & 0 & 1
\end{array}\right)\left(\begin{array}{c}
G^{ \pm} \\
\tilde{\phi}^{ \pm} \\
\hat{H}^{ \pm}
\end{array}\right) \\
& \left(\begin{array}{c}
\phi_{1}^{I} \\
\phi_{2}^{I} \\
\phi_{3}^{I}
\end{array}\right)=\left(\boldsymbol{x}_{12}^{+}, \boldsymbol{x}_{12}^{-}, \boldsymbol{x}_{3}\right)\left(\begin{array}{c}
G^{0} \\
G^{\prime 0} \\
A
\end{array}\right), \\
& \left(\begin{array}{c}
\phi_{1}^{R} \\
\phi_{2}^{R} \\
\phi_{3}^{R}
\end{array}\right)=R_{H}\left(\begin{array}{c}
H_{1} \\
H_{2} \\
H_{3}
\end{array}\right),
\end{aligned}
$$$$
=\left(\begin{array}{ccc}
c_{\beta} & s_{\beta} & 0 \\
-s_{\beta} & c_{\beta} & 0 \\
0 & 0 & 1
\end{array}\right)\left(\begin{array}{ccc}
1 & 0 & 0 \\
0 & c_{\theta_{H}} & -s_{\theta_{H}} \\
0 & s_{\theta_{H}} & c_{\theta_{H}}
\end{array}\right)\left(\begin{array}{c}
G^{ \pm} \\
H^{ \pm} \\
\hat{H}^{ \pm}
\end{array}\right),
$$

where $G^{ \pm}, G^{0}$, and $G^{\prime 0}$ are the NG bosons which are absorbed into the longitudinal component of $W^{ \pm}, Z$, and $Z^{\prime}$, respectively. In Eq. (B4), $R_{H}$ is the $3 \times 3$ orthogonal matrix which can be expressed by three independent mixing angles. These mixing angles are determined from the mass matrix for the $C P$-even Higgs bosons given in Eq. (B14). In Eq. (B3), $\boldsymbol{x}_{12}^{ \pm} \equiv\left(\boldsymbol{x}_{1} \pm \boldsymbol{x}_{2}\right) /\left|\boldsymbol{x}_{12}^{ \pm}\right|$and $\boldsymbol{x}_{3}$ are three component vectors defined as

$$
\boldsymbol{x}_{1}^{T}=\left(-\frac{v}{V} c_{\beta}\left(1+\frac{v^{2}}{V^{2}} c_{\beta}^{2}\right)^{-1 / 2}, 0,\left(1+\frac{v^{2}}{V^{2}} c_{\beta}^{2}\right)^{-1 / 2}\right),
$$

$$
\boldsymbol{x}_{2}^{T}=\left(c_{\beta},-s_{\beta}, 0\right),
$$

$$
\begin{aligned}
\boldsymbol{x}_{3}^{T}= & \left(s_{\beta}\left(1+\frac{v^{2}}{V^{2}} s_{\beta}^{2} c_{\beta}^{2}\right)^{-1 / 2}, c_{\beta}\left(1+\frac{v^{2}}{V^{2}} s_{\beta}^{2} c_{\beta}^{2}\right)^{-1 / 2},\right. \\
& \left.\left(1+\frac{V^{2}}{v^{2} s_{\beta}^{2} c_{\beta}^{2}}\right)^{-1 / 2}\right) .
\end{aligned}
$$

The squared masses of physical Higgs bosons and the mixing angle $\theta_{H}$ are given by

$m_{H^{ \pm}}^{2}=c_{\theta_{H}}^{2}\left(\mathcal{M}_{H^{ \pm}}^{2}\right)_{11}+s_{\theta_{H}}^{2}\left(\mathcal{M}_{H^{ \pm}}^{2}\right)_{22}+s_{2 \theta_{H}}\left(\mathcal{M}_{H^{ \pm}}^{2}\right)_{12}$,

$m_{\hat{H}^{ \pm}}^{2}=s_{\theta_{H}}^{2}\left(\mathcal{M}_{H^{ \pm}}^{2}\right)_{11}+c_{\theta_{H}}^{2}\left(\mathcal{M}_{H^{ \pm}}^{2}\right)_{22}-s_{2 \theta_{H}}\left(\mathcal{M}_{H^{ \pm}}^{2}\right)_{12}$, 


$$
\begin{gathered}
m_{A}^{2}=\frac{\sqrt{2} \mu V}{s_{2 \beta}}\left(1+\frac{v^{2}}{V^{2}} s_{\beta}^{2} c_{\beta}^{2}\right), \\
m_{H_{a}}^{2}=\left(R_{H}^{T} \mathcal{M}_{H}^{2} R_{H}\right)_{a a}, \quad(a=1,2,3), \\
\tan 2 \theta_{H}=\frac{2\left(\mathcal{M}_{H^{ \pm}}^{2}\right)_{12}}{\left(\mathcal{M}_{H^{ \pm}}^{2}\right)_{11}-\left(\mathcal{M}_{H^{ \pm}}^{2}\right)_{22}} .
\end{gathered}
$$

We can identify $m_{H_{1}}$ as the mass of the discovered Higgs boson, $125 \mathrm{GeV}$. The mass matrices $\mathcal{M}_{H^{ \pm}}^{2}$ and $\mathcal{M}_{H}^{2}$ are calculated as

$$
\mathcal{M}_{H^{ \pm}}^{2}=\left(\begin{array}{cc}
\frac{v^{2} \rho_{12}}{2}+\frac{\sqrt{2} V \mu}{s_{2 \beta}} & \frac{v V \xi_{1}}{2} \\
& m_{4}^{2}+\frac{V^{2}\left(\lambda_{34}+\rho_{34}\right)+v^{2}\left(c_{\beta}^{2} \lambda_{14}+s_{\beta}^{2} \lambda_{24}\right)}{2}
\end{array}\right),
$$

In the above expressions, the lower-left elements are the same as the corresponding transposed elements.

Next, we present the mass formulas for the $Z_{2}^{\mathrm{rem}}$-odd scalar states, in which there are one pair of doubly charged, three pairs of singly charged, and one neutral complex scalar states. The squared mass of the doubly charged scalar bosons $\eta_{\ell}^{ \pm \pm}$is given by

$m_{\eta_{\ell}^{ \pm \pm}}^{2}=m_{4}^{2}+\frac{1}{2}\left[V^{2} \lambda_{34}+v^{2}\left(c_{\beta}^{2} \lambda_{14}+s_{\beta}^{2} \lambda_{24}+s_{\beta}^{2} \rho_{24}\right)\right]$.

The mass eigenstates of the singly charged and neutral states are defined as follows:

$$
\begin{aligned}
\left(\begin{array}{c}
\eta_{2}^{ \pm} \\
\eta_{3}^{ \pm} \\
\eta_{\ell}^{ \pm}
\end{array}\right)= & \frac{1}{\sqrt{1+\frac{v^{2} s_{\beta}^{2}}{V^{2}}}}\left(\begin{array}{ccc}
\frac{v}{V} s_{\beta} & 1 & 0 \\
-1 & \frac{v}{V} s_{\beta} & 0 \\
0 & 0 & 1
\end{array}\right)\left(\begin{array}{c}
G^{\prime \pm} \\
\tilde{\eta}^{ \pm} \\
\eta_{\ell}^{ \pm}
\end{array}\right) \\
= & \frac{1}{\sqrt{1+\frac{v^{2} s_{\beta}^{2}}{V^{2}}}}\left(\begin{array}{ccc}
\frac{v}{V} s_{\beta} & 1 & 0 \\
-1 & \frac{v}{V} s_{\beta} & 0 \\
0 & 0 & 1
\end{array}\right)\left(\begin{array}{ccc}
1 & 0 & 0 \\
0 & c_{\theta_{\eta}} & -s_{\theta_{\eta}} \\
0 & s_{\theta_{\eta}} & c_{\theta_{\eta}}
\end{array}\right) \\
& \times\left(\begin{array}{c}
G^{\prime \pm} \\
\eta^{ \pm} \\
\hat{\eta}^{ \pm}
\end{array}\right),
\end{aligned}
$$

$$
\left(\begin{array}{c}
\eta_{1}^{0} \\
\eta_{3}^{0 *}
\end{array}\right)=\frac{1}{\sqrt{1+\frac{v^{2} c_{\beta}^{2}}{V^{2}}}}\left(\begin{array}{cc}
\frac{v}{V} c_{\beta} & 1 \\
-1 & \frac{v}{V} c_{\beta}
\end{array}\right)\left(\begin{array}{c}
G_{Y}^{0} \\
\eta^{0 *}
\end{array}\right)
$$

where $G^{\prime \pm}$ and $G_{Y}^{0}$ are the NG bosons which are absorbed into the longitudinal component of $W^{\prime}$ and $Y$, respectively. The squared masses of the physical scalar bosons and the mixing angle $\theta_{\eta}$ are given by

$$
m_{\eta^{ \pm}}^{2}=c_{\theta_{\eta}}^{2}\left(\mathcal{M}_{\eta^{ \pm}}^{2}\right)_{11}+s_{\theta_{\eta}}^{2}\left(\mathcal{M}_{\eta^{ \pm}}^{2}\right)_{22}+s_{2 \theta_{\eta}}\left(\mathcal{M}_{\eta^{ \pm}}^{2}\right)_{12},
$$

$$
m_{\hat{\eta}^{ \pm}}^{2}=s_{\theta_{\eta}}^{2}\left(\mathcal{M}_{\eta^{ \pm}}^{2}\right)_{11}+c_{\theta_{\eta}}^{2}\left(\mathcal{M}_{\eta^{ \pm}}^{2}\right)_{22}-s_{2 \theta_{\eta}}\left(\mathcal{M}_{\eta^{ \pm}}^{2}\right)_{12},
$$

$$
m_{\eta^{0}}^{2}=\frac{V^{2}}{2}\left(1+\frac{v^{2}}{V^{2}} c_{\beta}^{2}\right)\left(\rho_{13}+\sqrt{2} \tan \beta \frac{\mu}{V}\right)
$$

$$
\sin 2 \theta_{\eta}=\frac{2\left(\mathcal{M}_{\eta^{ \pm}}^{2}\right)_{12}}{m_{\eta^{ \pm}}^{2}-m_{\hat{\eta}^{ \pm}}^{2}}
$$

The mass matrix for the singly charged state $\mathcal{M}_{\eta^{ \pm}}^{2}$ is calculated as

$$
\mathcal{M}_{\eta^{ \pm}}^{2}=\left(\begin{array}{cc}
\frac{V^{2}}{2}\left(\rho_{23}+\frac{\sqrt{2} \mu}{V \tan \beta}\right)\left(1+\frac{v^{2}}{V^{2}} s_{\beta}^{2}\right) & \frac{v V \xi_{2} c_{\beta}}{2} \sqrt{1+\frac{v^{2}}{V^{2}} s_{\beta}^{2}} \\
\frac{v V \xi_{2} c_{\beta}}{2} \sqrt{1+\frac{v^{2}}{V^{2}} s_{\beta}^{2}} & m_{4}^{2}+\frac{V^{2} \lambda_{34}+v^{2} c_{\beta}^{2}\left(\lambda_{14}+\rho_{14}\right)+v^{2} s_{\beta}^{2} \lambda_{24}}{2}
\end{array}\right) .
$$




\section{APPENDIX C: FORMULAS FOR LEPTON FLAVOR VIOLATING DECAYS OF CHARGED LEPTONS}

We give the expressions for $a_{L, R}$ and $b_{L, R}$ appearing in the branching ratios of $\ell_{i} \rightarrow \ell_{j} \gamma$ and $\mu \rightarrow 3 e$ given in Eqs. (26) and (27), respectively. They can be separately expressed as

$$
a_{L, R}=\sum_{\varphi} a_{L, R}^{\varphi}, \quad b_{L, R}=\sum_{\varphi} b_{L, R}^{\varphi}
$$

where $a_{L, R}^{\varphi}$ and $b_{L, R}^{\varphi}$ denote the $\varphi$-loop $\left(\varphi=\eta^{0}, \eta^{ \pm \pm}\right.$, and $\hat{H}^{ \pm}$) contribution to the amplitude for the LFV decays. Each of them is calculated as follows:

$$
\begin{aligned}
\left(a_{R}^{\eta^{0}}\right)_{i j}=\left(a_{L}^{\eta^{0}}\right)_{i j}= & \frac{1}{16 \pi^{2}} \sum_{k}\left[\frac{\left(h_{L}^{*}\right)_{k j}\left(h_{L}\right)_{k i}}{2 M_{k}^{2}} G_{2}\left(\frac{m_{\eta^{0}}^{2}}{M_{k}^{2}}\right)\right. \\
& \left.+\frac{\left(h_{L}^{*}\right)_{k j}\left(h_{R}\right)_{k i}}{M_{k} m_{\ell_{i}}} G_{1}\left(\frac{m_{\eta^{0}}^{2}}{M_{k}^{2}}\right)\right], \quad(\mathrm{C} 2) \\
\left(a_{R}^{\eta^{ \pm \pm}}\right)_{i j}= & -\frac{1}{4 \pi^{2}} \sum_{k}\left(W^{T} F^{*}\right)_{k j}\left(W^{T} F\right)_{k i}\left[\frac{1}{2 M_{k}^{2}} G_{2}\left(\frac{m_{\eta^{ \pm \pm}}^{2}}{M_{k}^{2}}\right)\right. \\
& \left.+\frac{1}{m_{\eta^{ \pm \pm}}^{2}} G_{2}\left(\frac{M_{k}^{2}}{m_{\eta^{ \pm \pm}}^{2}}\right)\right], \quad(\mathrm{C} 3) \\
& \left(a_{R}^{\hat{H}^{ \pm}}\right)_{i j}=-\frac{1}{4 \pi^{2}} \frac{1}{12 m_{\hat{H}^{ \pm}}^{2}} \sum_{k} F_{k j}^{*} F_{k i}, \quad(\mathrm{C} 4) \\
\left(b_{L, R}^{\eta^{0}}\right)_{i j}= & -\frac{1}{12 \pi^{2}} \sum_{k} \frac{\left(h_{L, R}^{*}\right)_{k j}\left(h_{L, R}\right)_{k i}}{8 M_{k}^{2}} G_{3}\left(\frac{m_{\eta^{0}}^{2}}{M_{k}^{2}}\right), \quad(\mathrm{C} 6)
\end{aligned}
$$

[1] M. Singer, J. W. F. Valle, and J. Schechter, Canonical neutral current predictions from the weak electromagnetic gauge group $\mathrm{SU}(3) \times u(1)$, Phys. Rev. D 22, 738 (1980).

[2] J. W. F. Valle and M. Singer, Lepton number violation with quasi-Dirac neutrinos, Phys. Rev. D 28, 540 (1983).

[3] P. H. Frampton, Chiral Dilepton Model and the Flavor Question, Phys. Rev. Lett. 69, 2889 (1992).

[4] A. Zee, A theory of Lepton number violation, neutrino majorana mass, and oscillation, Phys. Lett. 93B, 389 (1980); Erratum, Phys. Lett. 95B, 461 (1980).

[5] A. Zee, Quantum numbers of Majorana neutrino masses, Nucl. Phys. B264, 99 (1986).

$$
\begin{aligned}
&\left(b_{L}^{\eta^{ \pm \pm}}\right)_{i j}= \frac{1}{12 \pi^{2}} \sum_{k}\left(W^{T} F\right)_{k j}^{*}\left(W^{T} F\right)_{k i}\left[\frac{1}{2 M_{k}^{2}} G_{3}\left(\frac{m_{\eta^{ \pm \pm}}^{2}}{M_{k}^{2}}\right)\right. \\
&+\left.\frac{1}{m_{\eta^{ \pm \pm}}^{2}} G_{4}\left(\frac{M_{k}^{2}}{m_{\eta^{ \pm \pm}}^{2}}\right)\right] \\
&\left(b_{L}^{\hat{H}^{ \pm}}\right)_{i j}= \frac{1}{12 \pi^{2}} \frac{1}{6 m_{\hat{H}^{ \pm}}^{2}} \sum_{k} F_{k j}^{*} F_{k i}, \\
&\left(b_{R}^{\eta^{ \pm \pm}}\right)_{i j}=\left(b_{R}^{\hat{H}^{ \pm}}\right)_{i j}=0
\end{aligned}
$$

where $h_{L}$ and $h_{R}$ are the coefficients of the $\bar{E}_{R}^{\prime} \ell_{L}^{\prime} \eta^{0 *}$ and $\bar{E}_{R}^{\prime} \ell_{R}^{\prime} \eta^{0 *}$ vertex, respectively, given as

$$
\begin{aligned}
& h_{L}=\frac{\sqrt{2}}{\sqrt{1+v^{2} c_{\beta}^{2} / V^{2}}} \frac{v}{V^{2}} c_{\beta} M_{E}^{\mathrm{diag}} W^{\dagger}, \\
& h_{R}=\frac{\sqrt{2}}{\sqrt{1+v^{2} c_{\beta}^{2} / V^{2}}} \frac{1}{v} W^{\dagger} M_{e}^{\mathrm{diag}} .
\end{aligned}
$$

The loop functions $G_{1,2,3,4}$ are given by

$$
\begin{gathered}
G_{1}(x)=\frac{1-4 x+3 x^{2}-2 x^{2} \ln x}{2(1-x)^{3}}, \quad(\mathrm{C} 11) \\
G_{2}(x)=\frac{1-6 x+3 x^{2}+2 x^{3}-6 x^{2} \ln x}{6(1-x)^{4}}, \quad(\mathrm{C} 12) \\
G_{3}(x)=\frac{-7+36 x-45 x^{2}+16 x^{3}+18 x^{2} \ln x-12 x^{3} \ln x}{6(1-x)^{4}}, \\
G_{4}(x)=\frac{2-9 x+18 x^{2}-11 x^{3}+6 x^{3} \ln x}{6(1-x)^{4}} .
\end{gathered}
$$

[6] K. S. Babu, Model of "calculable" Majorana neutrino masses, Phys. Lett. B 203, 132 (1988).

[7] T. P. Cheng and L.-F. Li, Neutrino masses, mixings and oscillations in $\mathrm{SU}(2) \times \mathrm{U}(1)$ models of electroweak interactions, Phys. Rev. D 22, 2860 (1980).

[8] L. M. Krauss, S. Nasri, and M. Trodden, A model for neutrino masses and dark matter, Phys. Rev. D 67, 085002 (2003).

[9] E. Ma, Verifiable radiative seesaw mechanism of neutrino mass and dark matter, Phys. Rev. D 73, 077301 (2006).

[10] S. M. Boucenna, S. Morisi, and J. W. F. Valle, Radiative neutrino mass in 3-3-1 scheme, Phys. Rev. D 90, 013005 (2014). 
[11] H. Okada, N. Okada, and Y. Orikasa, Radiative seesaw mechanism in a minimal 3-3-1 model, Phys. Rev. D 93, 073006 (2016).

[12] R. M. Fonseca and M. Hirsch, Lepton number violation in 331 models, Phys. Rev. D 94, 115003 (2016).

[13] A. C. B. Machado, P. Pasquini, and V. Pleitez, Zee and Zee-Babu mechanisms in the minimal 331 model, arXiv: 1810.02817.

[14] S. K. Kang, O. Popov, R. Srivastava, J. W. F. Valle, and C. A. Vaquera-Araujo, Scotogenic dark matter stability from gauged matter parity, Phys. Lett. B 798, 135013 (2019).

[15] J. Leite, O. Popov, R. Srivastava, and J. W. F. Valle, A theory for scotogenic dark matter stabilised by residual gauge symmetry, Phys. Lett. B 802, 135254 (2020).

[16] P. V. Dong, H. T. Hung, and T. D. Tham, 3-3-1-1 model for dark matter, Phys. Rev. D 87, 115003 (2013).

[17] P. V. Dong, D. T. Huong, F. S. Queiroz, and N. T. Thuy, Phenomenology of the 3-3-1-1 model, Phys. Rev. D 90, 075021 (2014).

[18] P. V. Dong, Unifying the electroweak and B-L interactions, Phys. Rev. D 92, 055026 (2015).

[19] K. Hasegawa, C.S. Lim, and K. Ogure, Escape from washing out of baryon number in a two zero texture general Zee model compatible with the LMA-MSW solution, Phys. Rev. D 68, 053006 (2003).

[20] X.-G. He, Is the Zee model neutrino mass matrix ruled out?, Eur. Phys. J. C 34, 371 (2004).

[21] D. Fregolente and M. D. Tonasse, Selfinteracting dark matter from an $\mathrm{SU}(3)(\mathrm{L}) \times \mathrm{U}(1)(\mathrm{N})$ electroweak model, Phys. Lett. B 555, 7 (2003).

[22] H. N. Long and N. Q. Lan, Sel-finteracting dark matter and Higgs bosons in the $\mathrm{SU}(3)(\mathrm{C}) \times \mathrm{SU}(3)(\mathrm{L}) \times \mathrm{U}(1)(\mathrm{N})$ model with right-handed neutrinos, Europhys. Lett. 64, 571 (2003).

[23] M. Ozer, $S U(3)-L \times U(1)-x$ model of the electroweak interactions without exotic quarks, Phys. Rev. D 54, 1143 (1996).

[24] Ya. B. Zeldovich, I. Yu. Kobzarev, and L. B. Okun, Cosmological consequences of the spontaneous breakdown of discrete symmetry, Zh. Eksp. Teor. Fiz. 67, 3 (1974) [Sov. Phys. JETP 40, 1 (1974)].

[25] T. W. B. Kibble, Topology of cosmic domains and strings, J. Phys. A 9, 1387 (1976).

[26] H. E. Haber and G. L. Kane, The search for supersymmetry: Probing physics beyond the standard model, Phys. Rep. 117, 75 (1985).

[27] J. Mrazek, A. Pomarol, R. Rattazzi, M. Redi, J. Serra, and A. Wulzer, The other natural two Higgs doublet model, Nucl. Phys. B853, 1 (2011).

[28] S. De Curtis, L. Delle Rose, S. Moretti, and K. Yagyu, A concrete composite 2-Higgs doublet model, J. High Energy Phys. 12 (2018) 051.

[29] S. De Curtis, L. Delle Rose, S. Moretti, and K. Yagyu, Supersymmetry versus compositeness: 2HDMs tell the story, Phys. Lett. B 786, 189 (2018).

[30] G. R. Dvali and G. Senjanovic, Is there a Domain Wall Problem?, Phys. Rev. Lett. 74, 5178 (1995).

[31] G. R. Dvali, A. Melfo, and G. Senjanovic, Nonrestoration of spontaneously broken $P$ and $C P$ at high temperature, Phys. Rev. D 54, 7857 (1996).
[32] J. Preskill, S. P. Trivedi, F. Wilczek, and M. B. Wise, Cosmology and broken discrete symmetry, Nucl. Phys. B363, 207 (1991).

[33] F. Riva, Low-scale leptogenesis and the domain wall problem in models with discrete flavor symmetries, Phys. Lett. B 690, 443 (2010).

[34] S. Weinberg, Gauge and global symmetries at high temperature, Phys. Rev. D 9, 3357 (1974).

[35] T. Blank and W. Hollik, Precision observables in $\operatorname{SU}(2) \times$ U(1) models with an additional Higgs triplet, Nucl. Phys. B514, 113 (1998).

[36] S. Kanemura and K. Yagyu, Radiative corrections to electroweak parameters in the Higgs triplet model and implication with the recent Higgs boson searches, Phys. Rev. D 85, 115009 (2012).

[37] H. Okada, N. Okada, Y. Orikasa, and K. Yagyu, Higgs phenomenology in the minimal $S U(3)_{L} \times U(1)_{X}$ model, Phys. Rev. D 94, 015002 (2016).

[38] J. Herrero-Garcia, T. Ohlsson, S. Riad, and J. Wiren, Full parameter scan of the Zee model: Exploring Higgs lepton flavor violation, J. High Energy Phys. 04 (2017) 130.

[39] T. Nomura and K. Yagyu, Zee model with flavor dependent Global $U(1)$ symmetry, J. High Energy Phys. 10 (2019) 105.

[40] K. S. Babu, P. S. Bhupal Dev, S. Jana, and A. Thapa, Nonstandard interactions in radiative neutrino mass models, J. High Energy Phys. 03 (2020) 006.

[41] J. Hisano, T. Moroi, K. Tobe, and M. Yamaguchi, Lepton flavor violation via right-handed neutrino Yukawa couplings in supersymmetric Standard Model, Phys. Rev. D 53, 2442 (1996).

[42] A. M. Baldini et al. (MEG Collaboration), Search for the lepton flavour violating decay $\mu^{+} \rightarrow \mathrm{e}^{+} \gamma$ with the full dataset of the MEG experiment, Eur. Phys. J. C 76, 434 (2016).

[43] B. Aubert et al. (BABAR Collaboration), Searches for Lepton Flavor Violation in the Decays $\tau \pm \rightarrow e \pm \gamma$ and $\tau \pm \rightarrow \mu \pm \gamma$, Phys. Rev. Lett. 104, 021802 (2010).

[44] U. Bellgardt et al. (SINDRUM Collaboration), Search for the decay $\mu^{+} \rightarrow e^{+} e^{+} e^{-}$, Nucl. Phys. B299, 1 (1988).

[45] P. F. de Salas, D. V. Forero, C. A. Ternes, M. Tortola, and J. W. F. Valle, Status of neutrino oscillations 2018: $3 \sigma$ hint for normal mass ordering and improved $C P$ sensitivity, Phys. Lett. B 782, 633 (2018).

[46] N. Aghanim et al. (Planck Collaboration), Planck 2018 results. VI. Cosmological parameters, arXiv:1807.06209.

[47] H.-Y. Cheng and C.-W. Chiang, Revisiting scalar and pseudoscalar couplings with nucleons, J. High Energy Phys. 07 (2012) 009.

[48] E. Aprile et al. (XENON Collaboration), Dark Matter Search Results from a One Ton-Year Exposure of XENON1T, Phys. Rev. Lett. 121, 111302 (2018).

[49] S. Kanemura, S. Matsumoto, T. Nabeshima, and N. Okada, Can WIMP dark matter overcome the nightmare scenario?, Phys. Rev. D 82, 055026 (2010).

[50] M. Escudero, A. Berlin, D. Hooper, and M.-X. Lin, Toward (finally!) ruling out $\mathrm{Z}$ and Higgs mediated dark matter models, J. Cosmol. Astropart. Phys. 12 (2016) 029.

[51] G. Arcadi, M. Dutra, P. Ghosh, M. Lindner, Y. Mambrini, M. Pierre, S. Profumo, and F. S. Queiroz, The waning of the WIMP? A review of models, searches, and constraints, Eur. Phys. J. C 78, 203 (2018). 
[52] S. Gori, C. Grojean, A. Juste, and A. Paul, Heavy Higgs searches: Flavour matters, J. High Energy Phys. 01 (2018) 108.

[53] M. Aoki, S. Kanemura, and K. Yagyu, Doubly-charged scalar bosons from the doublet, Phys. Lett. B 702, 355 (2011); Erratum, Phys. Lett. B 706, 495 (2012).

[54] R. D. Ball et al., Parton distributions with LHC data, Nucl. Phys. B867, 244 (2013).

[55] M. Aaboud et al. (ATLAS Collaboration), Search for doubly charged Higgs boson production in multi-lepton final states with the ATLAS detector using proton-proton collisions at $\sqrt{s}=13$ TeV, Eur. Phys. J. C 78, 199 (2018).

[56] M. Aoki, S. Kanemura, and H. Yokoya, Reconstruction of inert doublet scalars at the International Linear Collider, Phys. Lett. B 725, 302 (2013).
[57] A. Belyaev, G. Cacciapaglia, I. P. Ivanov, F. Rojas-Abatte, and M. Thomas, Anatomy of the inert two Higgs doublet model in the light of the LHC and non-LHC dark matter searches, Phys. Rev. D 97, 035011 (2018).

[58] S. Kanemura, T. Kasai, G.-L. Lin, Y. Okada, J.-J. Tseng, and C. P. Yuan, Phenomenology of Higgs bosons in the Zee model, Phys. Rev. D 64, 053007 (2001).

[59] Q.-H. Cao, G. Li, K.-P. Xie, and J. Zhang, Searching for weak singlet charged scalar at the Large Hadron Collider, Phys. Rev. D 97, 115036 (2018).

[60] Q.-H. Cao, G. Li, K.-P. Xie, and J. Zhang, Searching for weak singlet charged scalars at lepton colliders, Phys. Rev. D 99, 015027 (2019). 